\title{
Sensitivity of hurricane track to cumulus parameterization schemes in the WRF model for three intense tropical cyclones: Impact of convective asymmetry.
}

\author{
TRISTAN J. SHEPHERD \\ School of Earth Sciences, University of Melbourne, Melbourne, \\ VICTORIA, AUSTRALIA \\ KEVIN J. WALSH* \\ School of Earth Sciences, University of Melbourne, Melbourne, \\ VICTORIA, AUSTRALIA
}

SubmitTed to Meteorology AND ATMOSPHERIC PHysics

[MARCH 2016]

*Corresponding author address: Kevin J. Walsh, School of Earth Sciences, University of Melbourne, Victoria 3010, Australia

E-mail: kevin.walsh@.unimelb.edu.au, Phone: +61-3-8344-6523, Fax: +61-3-8344-7761. 
This study investigates the effect of the choice of convective parameterisation (CP) scheme on the simulated tracks of three intense tropical cyclones (TCs), using the Weather

4 Research and Forecasting (WRF) model. We focus on diagnosing the competing influences 5 of large-scale steering flow, beta drift and convectively-induced changes in track, as 6 represented by four different CP schemes (Kain-Fritsch (KF), Betts-Miller-Janjic (BMJ), 7 Grell-3D (G-3), and the Tiedtke (TD) scheme). The sensitivity of the results to initial conditions, model domain size and shallow convection is also tested.

We employ a diagnostic technique from Chan et al. (2002) that separates the influence of the large-scale steering flow, beta drift and the modifications of the steering flow by the storm-scale convection. The combined effect of the steering flow and the beta drift causes TCs typically to move in the direction of the wavenumber-1 (WN-1) cyclonic potential vorticity tendency (PVT). In instances of asymmetrical TCs, the simulated TC motion does not necessarily match the motion expected from the WN-1 PVT due to changes in the convective pattern. In the present study, we test this concept in the WRF simulations and investigate whether if the diagnosed motion form the WN-1 PVT and the TC motion do not match, this can be related to the emerging evolution of changes in convective structure.

Several systematic results are found across the three cyclone cases. The sensitivity of TC track to initial conditions (the initialisation time and model domain size) is less than the sensitivity of TC track to changing the $\mathrm{CP}$ scheme. The simulated track is not overly sensitive to shallow convection in the $\mathrm{KF}, \mathrm{BMJ}$, and TD schemes, compared to the track difference between CP schemes. The G3 scheme, however, is highly sensitive to shallow convection being used. Furthermore, while agreement between the simulated TC track direction and the $\mathrm{WN}-1$ diagnostic is usually good, there are important periods of differences that are often associated with model-generated convective asymmetries. 


\section{Introduction}

Moist convection varies widely in scale but is important for driving global scale atmospheric processes. Deep convective clouds have the ability to influence atmospheric processes and tropical cyclones (TCs) are extreme examples of deep moist convection. Moist convection must be represented accurately in atmospheric models for successful representation of dynamical/physical processes. Since this convection comprises very smallscale mixtures of updrafts and downdrafts, it is computationally expensive to represent these processes directly. Thus these processes must be parameterized in order to achieve accurate representation of moist convection. Stensrud (2007) argues that the grid spacing needed to resolve individual convective elements is likely to be between 25 and $1000 \mathrm{~m}$ and thus the realistic computational requirements needed to run routinely true convective resolving simulations lie in the future. Currently, most research and operational TC models are able to successfully employ resolutions of about $1 \mathrm{~km}$ (at convection-permitting resolutions), which is still too coarse for accurate treatment of subgrid scale processes (e.g. updrafts and downdrafts in convection-resolving simulations).

Convection is often parameterized in models through a cumulus parameterization (CP) scheme. A CP scheme must account for the rate of subgrid precipitation, latent heat release and redistribution of the thermodynamic and dynamic variables (such as heat and moisture) in the vertical as a result of convection (e.g Kain and Fritsch 1990). There are indications that it is possible to switch off $\mathrm{CP}$ at grid resolutions less than $4 \mathrm{~km}$ and allow an explicit microphysics scheme to resolve cloud and precipitation processes (Weisman et al. 1997). Horizontal resolutions around $4 \mathrm{~km}$ are often termed the "grey area" because at this scale a parameterisation of deep convection may be problematic, as convection is partly resolved and partly subgrid. On scales of $10-50 \mathrm{~km}$, however, the model still requires a CP scheme to accurately represent the subgrid scale processes. Thus for multiply-nested 
mesoscale models it has become a recent practice to use $\mathrm{CP}$ on the coarse outer domain (e.g. $12 \mathrm{~km}$ resolution) and use explicit microphysics for high resolution inner nests (e.g. $4 \mathrm{~km}$ resolution and finer), as used in Davis and Bosart (2002). Many CP schemes have been developed or modified over the past three decades (e.g. Ooyama 1971; Kuo 1974; Arakawa and Schubert 1974; Fritsch and Chappell 1980; Bougeault 1985; Betts 1986; Betts and Miller 1986; Frank and Cohen 1987; Tiedtke 1989; Gregory and Rowntree 1990; Emanuel 1991; Kain and Fritsch 1990; 1993; Kain 2004; Grell 1993; Grell and Devenyi 2002; Grell et al. 2013). The problem of CP was previously reviewed by Frank (1983) and Molinari and Dudek (1992). Arakawa (2004) and Stensrud (2007) have presented the most recent review of the CP problem, while others (e.g. Nasrollahi et al. 2012) have provided updates on specific topics.

A number of studies have examined the effect of the representation of convection on TC track. Dengler and Reeder (1997) found that relatively minor changes to the CP can have a large effect on the motion of a vortex, by changing the structure of the upper-level vortex and thereby altering the steering currents across the storm vortex. Prater and Evans (2002) note that the depth of the vortex can be altered by the choice of the convection scheme, with a scheme that produces a more elevated heating profile thereby responding to a deeper layer of mean steering currents, thus altering the storm direction. Srinivas et al. (2007) and $\mathrm{Li}$ and $\mathrm{Pu}$ (2009) demonstrated the sensitivity of storm track to the choice of convection scheme and also to the specification of the boundary layer physics within the model. Nasrollahi et al. (2012) examined the sensitivity of track to various combinations of CP schemes and cloud microphysics parameterizations, finding in their case that the Betts-Miller-Janjic parameterization gave the most accurate track. Sun et al. (2014) also showed the relationship between upper-level heating caused by differences in $\mathrm{CP}$ and the resulting changes in the steering flow of the storm through heating-induced changes in the geopotential height. 
The role of shallow convection in hurricane track and intensity prediction has also been explored. Shallow convection influences the boundary layer depth, temperature, moisture, cloud cover and winds (Bretherton et al. 2004; Kain 2004; Wang et al. 2004a,b; de Szoeke et al. 2006) and provides a pre-condition for deep convection to occur. The shallow component of a $\mathrm{CP}$ scheme will moisten the region above the boundary layer (up to about $600 \mathrm{hPa}$ ) and will modify the flow coming into the TC environment (Jimy Dudhia Pers. Comm., 2014.). Therefore, we should expect TC track sensitivity between simulations that use or do not use shallow convection, and between the use of different schemes, as each CP scheme represents shallow convection differently (see Table 1). In the present study, we aim to test this by showing track comparisons between simulations using no shallow convection and full convective cases.

In previous studies, Emanuel (1989) used an axisymmetric model to show that shallow convective clouds help to prevent overheating in the outer regions of a TC. In addition, low $\theta_{e}$ air is imported into the subcloud layer, which helps to cool the vortex, and leads to subsequent decay. In a 3D TC model developed by Zhu et al. (2001), Zhu and Smith (2002) explore the impact of shallow convection on the dynamics of TC intensification. They showed how shallow convection was able to stabilise the vortex core region, reducing the deep convective mass flux and buoyancy due to decreased rates of heating and drying in the troposphere. The diminished buoyancy in the vortex core decreased the vortex intensification rate. Using the Florida State University Global Spectral Model (Krishnamurti et al. 1998), O’Shay and Krishnamurti (2004) showed incremental improvement in TC track forecasts when shallow convection was included in the simulations. In a revision of convection and vertical diffusion schemes in the NCEP GFS model (Environmental Modeling Center, 2003), Han and Pan (2011) showed that using a new shallow convection scheme with a mass flux parameterisation, a decrease in TC track forecast errors resulted. This was attributed to an 
improvement in wind profiles, driven by heating throughout the convection layers, rather than cooling above and heating below, which the old turbulent-diffusion based shallow scheme produced. Torn and Davis (2012) showed an improvement in TC track forecasts when using the Tiedtke CP scheme compared to the Kain-Fritsch scheme. They stated the Tiedtke scheme allows for more appropriate treatment of oceanic shallow convection due to a more active shallow convection scheme than that in Kain-Fritsch. There was a $1 \mathrm{~K}$ temperature bias at $700 \mathrm{hPa}$ in the Kain-Fritsch simulations not present in the Tiedtke simulations, attributed to a lack of shallow convection in Kain-Fritsch. This generated horizontal temperature gradients associated with the wind biases affecting the TC tracks simulated with the Kain-Fritsch scheme.

While it is clear that differences in the simulation of convection can cause differences in track, here we present a detailed analysis of the effect of the choice of $\mathrm{CP}$ scheme on the simulated tracks of three intense tropical cyclones. Our focus will be on diagnosing the competing influences of large-scale steering flow, beta drift and convectively-induced changes in track, as represented by four different CP schemes. The sensitivity of the results to initial conditions, model domain size and shallow convection is also tested. The Weather Research and Forecasting (WRF) model (Skamarock et al. 2008) is used in this study. The WRF model is suitable for simulating hurricanes because of its full physics and 3D grid structure. While WRF has been widely employed for hurricane research (e.g. Davis et al. 2008; Hill and Lackmann 2009; Fovell et al. 2009; Cao et al. 2011; Kumar et al. 2011; Kieu et al. 2012; Cha and Wang 2013), sensitivity studies using it to examine the impact of CP differences on hurricane track and intensity are few. We present a range of WRF simulations from one North American case, one North West Pacific case and one Australian case. We find that sensitivity of TC track to initial conditions (initialisation time and model domain size) is considerably less than the sensitivity of TC track to the changing of $\mathrm{CP}$ scheme; the 
126 TC track is not particularly sensitive to shallow convection in the Kain-Fritsch, Betts-Miller-

127 Janjic and Tiedtke schemes (see section 2 for descriptions of these parameterizations), but TC track is highly sensitive in the G3 scheme; and the G3 simulation with shallow convection consistently performed poorly across all three cyclone cases. Section 2 outlines the methods used. The cases are outlined in section 3. The results are presented in section 4 and discuss track and intensity sensitivity to changes in $\mathrm{CP}$ scheme, and the physical mechanisms for this sensitivity. We then summarise this work by placing it in context of other studies on TC motion and CP sensitivity.

\section{Methodology}

\subsection{Model and domain specification}

The version of WRF used here is v3.3.1 (Skamarock et al. 2008). An experimental design is used to eliminate other factors which may cause model simulation sensitivity to something other than CP specification (e.g. initial condition error). Two criteria were selected to test the initial condition sensitivity: initialization time and model domain size. To test the sensitivity of the track simulation to initial conditions, we conduct the same simulations but initialised 6 hours later. To estimate the sensitivity due to lateral boundary conditions, we conduct the same simulations maintaining the inner-nest dimensions, but use a smaller outer coarse domain (see Fig. 1). For these tests, all physics parameterisations and other model settings remain identical and only the $\mathrm{CP}$ is changed.

In order to make some general conclusions about the CP schemes, a selection of three, well-simulated category 4 or 5 hurricanes from different basins are chosen as case studies. These storms were Rita (2005), Megi (2010) and Yasi (2011) (see section 3). There are a total of 16 simulations for each cyclone case. Four CPs are tested (and summarised in Table 1): Kain-Fritsch (KF) (Kain and Fritsch 1990; 1993; Kain 2004), Betts-Miller-Janjic (BMJ) 
(Betts 1986; Betts and Miller 1986; Janjic 1994), Grell-3D (G3) (Grell and Devenyi 2002), and Tiedtke (TD) (Tiedtke 1989; Zhang et al. 2011). Simulations which are denoted ' +6 ' and 'dom2' explore the initial condition uncertainty: the ' +6 ' simulations are initialised 6 hours later and the 'dom2' simulations use a different domain size. A separate set of experiments denoted 'NO_SHALLOW' (hereafter NS) explore the sensitivity of track and intensity when the shallow convection component of each $\mathrm{CP}$ scheme is turned off. The purpose of these tests is to ascertain the role of shallow convection and whether the shallow convection processes modify the environment, or whether it is the deep convection controlling the large scale. Note that in the case of the G3 scheme, shallow convection is not included by default; the user must activate a switch to run shallow convection with this scheme. Thus, simulations denoted G3_NS are the default configuration of the scheme, whereas those denoted G3 will have shallow convection included.

The physics options used in all the experiments were the MYJ PBL scheme (Mellor and Yamada 1982; Janjic 1990; Janjic 2001), the Eta microphysics scheme (Rogers et al. 2001), the rapid radiative transfer model (RRTM) for longwave radiation (Mlawer et al. 1997), the Dudhia scheme for shortwave radiation (Dudhia 1989), and the Monin-Obukhov (Janjic Eta) surface layer scheme (Janjic 1996; 2001). The domain configuration for the standard simulations (dom1) is shown in Fig. 1. The parent (i.e. outer) domain has a resolution of $12 \mathrm{~km}$ and $423 \times 324$ grid points and the inner movable nests are $4 \mathrm{~km}$ and 1.33 km with $201 \times 201$ and $240 \times 240$ grid points respectively. Note that the 'dom2' experiments have the same nest dimensions, but an outer domain of 380 × 280 grid points. The model has 33 vertical levels with a top of $50 \mathrm{hPa}$. Initial and boundary conditions for the large-scale atmospheric fields were derived from $1^{\circ}$ x $1^{\circ}$ NCEP Global Final Analysis (FNL) data (National Centers for Environmental Prediction/National Weather Service/NOAA/U.S. Department of Commerce, 2000). The model was updated with FNL data every 6 hours. Nest 
positions were updated every 15 minutes using an automatic vortex-following algorithm which tracked the centre of the vortex at $750 \mathrm{hPa}$. One-way nesting is used, and $\mathrm{CP}$ is not used in the inner-nests.

\subsection{Diagnostic methods}

A diagnostic technique is employed that separates the influence of large-scale steering flow, beta drift and the modifications of the steering flow by storm-scale convection. For the definition of the steering flow, we use the deep-layer mean flow between $850 \mathrm{hPa}$ and 200 hPa (e.g. based on Chan and Gray 1982).

The motion of a tropical cyclone deviates from the direction of the large-scale steering flow due to "beta-drift", caused by the interaction between the vortex and the gradient of the Coriolis force (Holland 1983; Chan and Williams 1987). Wu and Wang (2000) showed that the combined effect of the steering flow and the beta drift causes tropical cyclones typically to move in the direction of the wavenumber-1 (WN-1) cyclonic potential vorticity tendency (PVT). Wu and Wang (2000) used idealised symmetric baroclinic TCs in their study. For simulations of asymmetrical TCs, however, Chan et al. (2002) showed that the diagnosed WN-1 PVT and the simulated TC motion may not match exactly, as changes in the convective pattern can lead to track oscillations and/or track changes that differ from the expected direction indicated by the PVT. In this circumstance, the motion of the TC has not yet responded to an environmental change in the PV forcing. In the present study, we test this concept in the WRF simulations and investigate whether if the diagnosed motion from the WN-1 PVT and the TC motion do not match, this can be related to the emerging evolution of changes in convective structure (see also Chan and Kepert 2010). To our knowledge, this is the first time that this diagnostic has been applied to simulations using the WRF model. 

and is only briefly outlined here. In order to calculate the PVT, a 6 hour average of PV is taken around time 1 and the same is then done for time 2. The $\mathrm{WN}-1$ component is then extracted for each averaged field (i.e. PV at time 1 and PV at time 2). The PVT $\left(\frac{\partial q_{1}}{\partial t}\right)$ is then calculated. Here, the time difference between PV (time1) and PV (time2) is 6 hours (this is chosen to minimise the effect of storm movement in the domain). TC motion vectors used in the analysis are calculated over a 12 hour period. The latitude and longitude values from the model-simulated track output data are used to determine the TC motion vectors. The steering current is calculated over a 12 hour period to match the TC motion time frame. Note that there is a difference between the time intervals over which the WN-1 PVT is calculated and the steering current time frame, but tests showed little difference between 12- and 6-hour average steering currents. In instances where we have studied specific periods of interest, we calculated the TC motion over 6 hours to match the WN-1 PVT (note that the 6- and 12-hour TC motion directions also showed minimal difference). Cyclone centred composites of $u$ and $v$ wind fields are first derived before having the deep layer mean method applied. The vortex is removed using a standard WN-1 technique (Wu and Wang (2000); Yuqing Wang, Pers. Comm., 2013) to obtain the environment flow with the TC vortex removed. The steering vector is then determined by calculating the mean over this environment flow.

\section{Cyclone Case Studies} January to 3 February 2011. Yasi was a category 4 cyclone on the Saffir-Simpson scale, and a 
IBTrACS data, the maximum 10-minute sustained wind speed Yasi attained was 110 knots (estimated) and the lowest atmospheric pressure was $929 \mathrm{hPa}$. Total damage was estimated at A $\$ 800$ million (Brisbane Times, 2011), with an estimate of a US\$3.54 billion cost to insurers, making Yasi the costliest storm to hit the Australian coast (Reuters, 2011).

\subsection{Hurricane Rita}

Hurricane Rita (Fig. 2) occurred in the north Atlantic hurricane basin in September 2005. Rita was a Saffir-Simpson category 5 cyclone which followed only one month after hurricane Katrina and was responsible for 120 deaths across four US states. Rita was the fourth most intense Atlantic hurricane ever recorded and the most intense hurricane ever in the Gulf of Mexico. The maximum 10-minute sustained wind speed was 153 knots and lowest atmospheric pressure was $895 \mathrm{hPa}$. Rita was a costly hurricane: over US\$12 billion in damage was reported (Knabb et al. 2006). Hurricane Rita is an important case to study due to its intensity.

\subsection{Super Typhoon Megi}

Super-typhoon Megi (Fig. 2) occurred in the western north Pacific hurricane basin in

October 2010. Megi was a Saffir-Simpson category 5 cyclone responsible for the deaths of 69 people in Luzon, Philippines and Yilan County, Taiwan. Megi was the most intense tropical cyclone since Bess in 1982 (for wind speed) and had the lowest atmospheric pressure in the Northwest Pacific Ocean since Vanessa in 1984. The maximum 10-minute sustained wind speed Megi attained was 159 knots and the lowest atmospheric pressure was $885 \mathrm{hPa}$. Megi was one of the costliest tropical cyclones to hit the Philippines, with damage of US\$107 million (National Disaster Risk Reduction and Management Council, 2010). 


\section{Results}

This section presents simulated cyclone track results to explore whether there is sensitivity to the $\mathrm{CP}$ scheme. Some consideration is also given to simulated intensity sensitivity. We then explain our track sensitivity findings by showing the physical mechanisms responsible for track sensitivity.

\subsection{Simulated cyclone track}

\subsubsection{Sensitivity to initial conditions}

Two criteria were selected to test initial condition sensitivity: initialization time and model domain size. The track results of these tests can be viewed in Fig. 2. We consider each cyclone case in turn by examining the landfall distance difference in $\mathrm{km}$ between each simulation. We employ this metric rather than the difference between simulated and observed tracks to assess the size of these effects compared with the sensitivity to the CP scheme rather than their impact on track quality, which is discussed later.

\subsubsection{Yasi}

The landfall difference from the control simulation for each $\mathrm{CP}$ scheme due to the change in initialization time (designated in the tables as "vs. + 6", column 2) varied between each scheme (Table 2). The overall average for all CP schemes was a difference of $59 \mathrm{~km}$ (column 2). In contrast, the average landfall difference between the $\mathrm{CP}$ schemes is $236 \mathrm{~km}$ (column 4). The tracks for storms initialised at 00Z (solid lines) and 6 hours later (06Z, dashed lines) are shown in Fig. 2a.

When the model domain size was changed (vs DOM2, column 3), the average landfall distance was $55 \mathrm{~km}$, still considerably smaller than the sensitivity to the $\mathrm{CP}$ scheme. For the DOM2 runs, the $\mathrm{CP}$ sensitivity average was $172 \mathrm{~km}$ (column 7), $64 \mathrm{~km}$ less than the $\mathrm{CP}$ sensitivity average for the experiments run on the original domain. The tracks for storms 
initialised at 00Z (solid lines) and for DOM2 (dashed lines) are shown in Fig. 2b. It is clear from both the data in Table 2 and Fig. 2 that the sensitivity of track to both changes in the initial conditions and domain specification is less than the sensitivity to the specification of CP.

\subsubsection{Rita}

For Rita (Table 3), similar behaviour is seen. The average landfall distance difference for the initialisation test was $124 \mathrm{~km}$ (column 2). As for the Yasi case, landfall distance difference was smaller when the model domain size was changed (an average of $89 \mathrm{~km}$ ). Unlike in Yasi, where track deviations at the start of the forecast explained the differences, in Rita the differences occur due to deviations towards the latter stages of its life cycle (see the tracks in Fig. 2d-e). Similarly to Yasi, the average landfall distance differences due to changes in the $\mathrm{CP}$ scheme were considerably larger, $237 \mathrm{~km}$ for the original domain and 258 $\mathrm{km}$ for the DOM2 domain.

\subsubsection{Megi}

For Megi (Table 4), unlike the Yasi and Rita cases, when the domain size was changed the average difference increased (compare columns 2 and 3). Similarly to the Yasi case, the tracks tended to deviate in the early stages of their life cycle (Fig. $2 g-h$ ). Again, though, the sensitivity to the changes in the $\mathrm{CP}$ scheme was considerably larger than to changes in initial conditions or domain size. Both the $00 \mathrm{Z}(338 \mathrm{~km}$, column 4$)$ and $+6 \mathrm{Z}$ average $(388 \mathrm{~km}$, column 8$)$ are greater than the initial condition average $(95 \mathrm{~km}$ for $+6 \mathrm{Z}$, column 2, and $127 \mathrm{~km}$ for DOM2, column 3).

\subsubsection{Summary}

The simulated track is more sensitive to both changes in the initial conditions and domain specification than the sensitivity to the specification of $\mathrm{CP}$. The average landfall difference increased when the $\mathrm{CP}$ scheme was changed compared to the difference from 
initialization time or model domain size change.

\subsubsection{Sensitivity to shallow convection scheme}

Exploring the sensitivity to the use of shallow convection determines whether it is the deep or shallow component in CPs which modifies the surrounding synoptic environment and thus influences track more. Fig. $2 \mathrm{c}, 2 \mathrm{f}$, and $2 \mathrm{i}$ show tracks for simulations where the shallow convection component was switched off. These are compared to the tracks where both the deep and shallow operated (i.e. the normal use of CP). Each case is discussed with reference to the data in Tables $2-4$.

\subsubsection{Yasi}

The KF, BMJ and TD schemes have virtually identical tracks to their no shallow counterparts (Fig. 2c). Indeed, the landfall distance difference between each CP case and the NS equivalent is 15,14 and $37 \mathrm{~km}$ respectively (Table 2, Column 5). In contrast, the G3 simulations exhibited strong sensitivity to the use of shallow convection: the difference in landfall between G3 and G3_NS was $278 \mathrm{~km}$. This meant the average across CP schemes of $86 \mathrm{~km}$ is inflated by this outlier. When the scheme was run normally (G3_NS), the track was more in line with that of the KF and TD schemes (compare green dash with solid yellow and red track lines in Fig. 2c). When shallow convection was used (G3), the track followed a similar path to the BMJ.

\subsubsection{Rita}

A similar result to the Yasi case is observed with the Rita simulations. Here the $\mathrm{KF}+6$ v KF+6_NS, BMJ+6 v BMJ+6_NS and TD+6 v TD+6_NS simulations showed some small difference at landfall: 41, 57 and $65 \mathrm{~km}$ respectively (Table 3, column 5 and Fig. 2f). The same behaviour observed in the Yasi case was seen in the Rita G3 simulations, with a large difference between G3+6 and G3+6_NS of $287 \mathrm{~km}$ (Table 3, column 5). Once again, the G3+6_NS track is similar to the KF and TD tracks (compare green dash with red and yellow 
tracks in Fig. 2f), and the G3+6 is similar to the BMJ tracks at landfall. The average was 112 $\mathrm{km}$, which is also inflated by the large G3 simulation difference. In this case, the average was less than that for the $00 \mathrm{Z}$ initial condition comparison, but more than the average for the DOM2 simulations.

\subsubsection{Megi}

As in the other two cases, the same general result is found with the Megi simulations. When shallow convection is turned on, the TD simulation once again shows a slight difference at landfall $(100 \mathrm{~km})$, but the $\mathrm{KF}$ and BMJ runs are virtually in identical landfall positions (a difference of 10 and $2 \mathrm{~km}$ respectively), while the G3 simulations have a much larger difference of $343 \mathrm{~km}$ (Table 4, column 5), and the northward drift seen in G3 is not present in G3_NS (Fig. 2i). In this instance, turning on the shallow convection scheme in G3 has caused a large change to the TC track, causing it to deviate greatly from the observed track. Another relevant track observation is that the looping of the BMJ track is removed in BMJ_NS, but there is still a notable southwest drift in both compared with the observed track (black line). The average difference is $113 \mathrm{~km}$, again largely due to the large difference in the G3 simulations. This is slightly more than the difference between the $06 \mathrm{Z}$ initial condition simulations $(95 \mathrm{~km})$, but less than the difference in the DOM2 simulations $(127 \mathrm{~km})$.

\subsubsection{Summary}

The simulated track is not overly sensitive to shallow convection in the KF, BMJ, and TD schemes, compared to the track difference between CP schemes. This is a systematic result seen across all cyclone cases. The G3 scheme, however, is highly sensitive to the shallow convection being used. The reasons for the difference in the G3 simulations are investigated in later sections.

\subsubsection{Track sensitivity to $\mathrm{CP}$ scheme}

It is clear from the simulated track results and data in Tables $2-4$ that there is track 
sensitivity to CP shown across all cyclone cases. For the purposes of comparison, treating the

$\mathrm{KF}$ simulation as control (KF is widely used and studied in the literature, thus enabling a reference point for comparison with other studies), we are able to analyse the landfall distance difference between simulations as an example of this sensitivity. This is shown in Tables $2-4$ (column 4, under the heading ' $K F$ vs. other $C P s$ ' [+6 in the case of Rita]). The BMJ cases and the G3 simulation tend to be the ones which differ significantly in the Yasi and Rita cases. In contrast, in these cases the KF, TD and G3_NS simulations tend to be grouped more closely (e.g. Figs. 2c and 2f). The Megi case has much more track variability (e.g. Fig. 2i): here, the G3_NS and TD were close to each other, but the KF was not grouped with them. Physical reasons for the sensitivity are discussed in section 4.3.

\subsubsection{Comparison to observed best track}

One means to assess the performance of the schemes is to compare landfall position between the CP scheme and the observed best track. Due to the small number of TC cases shown here, it should be noted this does not necessarily reflect the overall performance of a scheme, which would need to be assessed by a statistical analysis of dozens of TC cases. Nevertheless, a very poor simulation of track or tropical cyclone structure would be an indication of performance even in a limited set of simulations. The data comparing the difference between the simulated landfall position to the observed landfall position is given in Table 5, along with an average difference for each simulation across the three cases. This takes the landfall difference between the simulated and observed for each simulation and averages the difference across all three cyclone cases. We can then break this down for individual scheme by averaging the four runs for each scheme (i.e. average of $\mathrm{KF}, \mathrm{KF}+6$, KF_NS, KF_DOM2 across all cyclone cases) to obtain an average landfall difference across all cyclone cases according to experiment group (note that the averages for G3 are affected by the Megi simulation not making landfall). From these values we note that G3, regardless 
of the Megi case not making landfall, still produced the largest average difference between the simulation and observed landfall $(318 \mathrm{~km})$ (Table 5, row 3). In contrast, the average for G3_NS was $144 \mathrm{~km}$ (Table 5, row 11). The TD case had a difference of only $86 \mathrm{~km}$ and this is reflected throughout the other TD runs, with an overall difference average (i.e. average of TD, TD+6, TD_NS, TD_DOM2) of only $146 \mathrm{~km}$, compared to KFs (245 km), BMJs (241 $\mathrm{km})$ and G3s $(246 \mathrm{~km})$. Obviously there is substantial variability for each specific simulation/scheme between cyclone cases. Some schemes may perform better for certain cases compared to other schemes, but the averaging does tend to indicate that the TD and G3_NS simulations were particularly consistent in having low track error across all three cases. G3, however, tended to produce both variability and larger errors across all three cases. $\mathrm{KF}$ and $\mathrm{BMJ}$ tended to be somewhere in the middle.

\subsection{Simulated cyclone intensity}

The emphasis of this study is on the sensitivity of simulated track, but some consideration must be given to intensity also. Without reasonable intensity prediction, track comparison becomes less meaningful. Different representation of $\mathrm{CP}$ can also lead to dissimilar TC intensity, which can affect the TC track. Simulated intensity is compared for the initial condition and shallow CP simulations for each case.

\subsubsection{Yasi}

Simulated pressure differences between the $00 \mathrm{Z}$ and $+06 \mathrm{Z}$ cases are shown in Fig. $3 \mathrm{a}-\mathrm{b}$. For each scheme, there is minimal separation between the $00 \mathrm{Z}$ and $+06 \mathrm{Z}$ counterpart over the first 24 hours, but later into the forecast minor changes emerge. At most times after 24 hours, the difference is less than $15 \mathrm{hPa}$ in all the cases, which reinforces the point regarding minimal sensitivity to initial conditions. Moreover, the variation between the schemes themselves, particularly the $\mathrm{KF}$ and $\mathrm{BMJ}$, is more pronounced than the intensity change from changing the initial time. This confirms previous findings for the tracks, where 
394

$\mathrm{CP}$ changes result in more sensitivity than changes in initial time. There is, however, little difference between the G3, TD and BMJ simulated intensity over most of the simulation period. The KF produced the deepest storm and subsequently is more separated from the other cases. Finally, in comparison to the observations, given no bogussing or other data assimilation was applied, the model does well to match intensity, especially when using the KF scheme.

The simulated wind speed is recalculated as a six hour moving average in order to observe more general trends (Fig. 3b). The wind speed rises gradually over the simulation period, consistent with a gradual spin up over the first 24 hours. The wind speed results mirror those for pressure. In comparison to observations, wind speed tends to be overpredicted in the first $24-36$ hours, which may be a result of initialisation error.

The comparison of the shallow and NS convection cases illustrates an identical response to that seen for the tracks. The simulated pressure difference between the shallow and NS cases is minimal, except in the G3 scheme, where G3 run with shallow convection (G3) produced a much weaker storm than G3_NS (Fig. 3c). The same result is reflected in the associated wind speed (Fig. 3d).

\subsubsection{Rita}

Simulated pressure (Fig. 3e) shows no significant differences between the $00 \mathrm{Z}$ and $+06 \mathrm{Z}$ cases, except minor separation (i.e. $5 \mathrm{hPa}$ ) between the G3 and G3+6 cases. Despite there being marked track sensitivity, sensitivity of simulated pressure to $\mathrm{CP}$ scheme is less than $20 \mathrm{hPa}$. The most significant observation here is that simulated pressure is not able to match the rapid intensification of Rita, which in the absence of bogussing, is likely a result of current model progress in parameterisation schemes, or other TC processes involved in rapid intensification, such as strong SST changes, or lack of coupling with an appropriate ocean model. Simulated wind speed, plotted as a six hour moving average (Fig. 3f) matches the 
findings for pressure. In contrast to Yasi, BMJ performed best here, with G3 weakest. The use of no shallow convection has negligible effect in Rita also. Simulated pressure difference (Fig. 3g) between the two types of run is insignificant. In this cyclone case, however, despite there being substantial track difference between the G3+6 and G3+6_NS, the simulated pressure does not differ to the same degree. Only after 72 hours do the pressures begin to deviate from each other, and even then it is less than $10 \mathrm{hPa}$ in difference. The six hour moving average for wind speed shows more variability between G3+6 and G3+6_NS (Fig. 3h), but this is in the latter stages of the simulation, which matches the same period for the pressure deviation. The other simulations (e.g. KF+6 vs. KF+6_NS; BMJ+6 vs. BMJ+6_NS; TD+6 vs. TD+6_NS) do not show this same separation trend as that seen between G3+6 and G3_NS+6, that is, when the +06Z is compared to the NS case, the other cases tend to show very minimal pressure and wind difference.

\subsubsection{Megi}

The simulated pressure for different initial conditions (Fig. 3i) shows the only case to exhibit sensitivity to initial time was G3: the G3+6 simulation is considerably weaker than the G3. There are also intensity differences between the schemes, with BMJ somewhat weaker than $\mathrm{KF}$ and TD. The weakening of BMJ after 48 hours is consistent with its looping track. The TD and KF cases reach an intensity of $930 \mathrm{hPa}$ before stabilising. None of the cases are able to match the rapid intensification observed after 60 hours (the G3 result is not meaningful given the track was so poor). Additionally, the wind speed intensity (Fig. 3j), confirms what is seen for pressure. Simulated intensity here, however, is much greater than the observed wind speeds. Megi took considerable time to intensify, whereas the model simulated pressure and wind speed intensity are both ahead of the observed values until after 72 hours.

The effect of running no shallow convection follows the trend in the other cyclone 
cases, where only the G3 case shows sensitivity. Simulated pressure (Fig. 3k) indicates no difference for TD and KF, minimal difference in BMJ, and a large separation beginning after 60 hours for G3 and G3_NS. While there is a large difference in G3 pressure, the same is not seen in the wind speed average (Fig. 31). This can be caused by changes in the pressure gradient or storm size.

\subsubsection{Summary}

For the cases, the intensity sensitivity resulting from changing the $\mathrm{CP}$ scheme is greater than the sensitivity from initial condition changes. This agrees with the simulated track result. The only case to show sensitivity to initial conditions was Megi for G3 and G3+6. In addition, the use of no shallow convection shows minimal difference to the simulations that used shallow convection, all except the G3 and G3_NS runs.

\subsection{Physical mechanisms for sensitivity to cumulus scheme} the differences between the $\mathrm{KF}$ and the BMJ simulations, and the G3 and G3_NS simulations. This is because $\mathrm{KF}$ and $\mathrm{BMJ}$ are physically different $\mathrm{CP}$ schemes, being essentially mass-flux and convective adjustment schemes respectively, consistently produced tracks which varied from each other. Furthermore, the track sensitivity between the G3 simulation, where the shallow component of the G3 scheme was switched on, and the G3_NS simulation, where shallow was switched off, was quite marked. Note that the TD simulations are not analysed further because the KF, G3_NS and TD tracks were similar, and with all of them being mass flux schemes, only KF was selected for comparison to BMJ.

\subsubsection{Yasi}

The KF and BMJ simulations produced considerably different modelling outcomes

(Fig. 2). While both simulations had a westward and poleward track similar to observations, the BMJ (solid blue) was displaced much further south-westward than KF (solid red). A 
similar comparison can be made between G3 (solid green line) and G3_NS (green dashed line) (Fig. 2c).

A comparison of the TC motion and steering vectors for KF (Fig. 4a) and BMJ (Fig. 4b) shows rather different motion between the schemes. Hours $12-24$ are a crucial period in 473 this study, as during this period KF tracks at $267^{\circ}$ (Fig. 4a,ii) while BMJ tracks at $237^{\circ}$ (Fig. 4b,ii), thereby leading to a substantial difference in position between the two after 24 hours. Another substantial deviation occurs over hours 36 - 48. At this particular time, KF is tracking at $248^{\circ}$ (Fig. 4a,iv), but BMJ is at $227^{\circ}$ (Fig. 4b,iv), leading to an even greater separation between the two tracks. For the remainder of the simulation, the two track directions are very similar, so the resulting difference between the two simulations is driven by the differences over hours $12-24$ and $36-48$. In addition, if we also consider steering, after 48 hours of simulation (e.g. onward from hour 48 of the simulation), the steering current vector mostly shows less angular difference from the motion vector in $\mathrm{KF}$, which indicates the beta effect is generally less than that in BMJ. The steering current magnitudes are not necessarily larger in BMJ; it is simply that there is a difference in their direction. A greater beta effect in BMJ (a function of differences in the TC motion vectors between $\mathrm{KF}$ and $\mathrm{BMJ}$, and the larger storm size in BMJ [not shown]) likely results in greater poleward motion. In this case, for a westward moving storm in the Southern Hemisphere, beta drift typically adds an additional southward component of motion to the steering flow (Holland 1984; Carr and Elsberry 1990).

A similar comparison can be made for the simulation outcome between G3 and

G3_NS (green solid and green dashed lines in Fig. 2c). The TC motion and steering vectors (Fig. 4c for G3 and Fig. 4d for G3_NS) show the differences between the two cases. There is a difference in the first 12 hours of simulation, which is negated after 24 hours because the two tracks are then in the same location. A key separation occurs between the hours of $36-$ 
48 (compare Figs. 4c,iv and 4d,iv), which is the main reason for the subsequent difference in simulated track outcome. At 36 hours, G3 turns to a heading of $239^{\circ}$, while G3_NS turns to a heading of $248^{\circ}$. The result is the G3 simulation moving the TC further south $\left(16.1{ }^{\circ} \mathrm{S}, 155\right.$ $\left.{ }^{\circ} \mathrm{E}\right)$ and G3_NS further north $\left(15.9{ }^{\circ} \mathrm{S}, 154.6{ }^{\circ} \mathrm{E}\right)$, respective to their positions at hour 36. Once the two tracks are separated the difference is maintained, with a motion difference of $10^{\circ}$ between the two cases over hours $48-60$ and $60-72$. The resulting difference in landfall position is driven by these subtle differences. In addition, if steering is considered, we note that the magnitude of the steering current in G3 increases relative to G3_NS over time (e.g. G3 is $0.8 \mathrm{~m} \mathrm{~s}^{-1}$ larger than G3_NS for 60 - 72). An angular difference between the TC motion and steering also develops in G3 that is not seen in G3_NS until hours $72-84$ (compare Fig. 4c,v-vii with 4d,v-vii). Like BMJ, a greater beta effect in G3 likely results in greater poleward motion compared to G3_NS, but convective effects cannot be ruled out, and will now be examined.

For the purposes of analysing the differences in the simulated track between the cases, we present a table assessing the simulated TC motion against the diagnosed motion derived from the WN-1 PVT analyses, the findings of which are given in Table $6-8$ as directions in degrees. In order to make a direct comparison, for the specific times analysed we calculate the TC motion over a 6 hour period. We here define "agreement" as cases where there is a small difference between the direction of TC motion and the WN-1 PVT diagnostic. To quantify these descriptions, in terms of the agreement between the WN-1 PVT and the simulated TC motion, if we take a typical storm speed of $15 \mathrm{~km} / \mathrm{h}\left(4.1 \mathrm{~m} \mathrm{~s}^{-1}\right)$ and the difference between the WN-1 PVT and the simulated TC motion was $30^{\circ}$, then there is a distance deviation of less than one-half of the actual distance travelled by the storm (note that we used the 6 hour TC motion speed). In such a situation we would quantify the agreement between the WN-1 PVT and simulated TC motion as fair. Good agreement would therefore 
be any deviation lower than this, and poor agreement anything substantially higher. In terms of whether a storm is strongly steered, we quantitatively define a strongly steered storm as one that has a steering current magnitude above the average distribution of the steering current magnitudes observed in these experiments. For the cases where the directional agreement between the two is not good, other dynamics, such as the convective structure, are studied to help explain why the directions may not match.

The overall conclusion of Tables 6-8 is that while agreement between the TC track direction and the $\mathrm{WN}-1$ diagnostic is usually good, there are important periods of disagreement. In order to explain the track difference between $\mathrm{CP}$ schemes, we choose analysis times where there is a significant track difference. For KF and BMJ, we identified this as between hours $12-24$ (Fig. 4a,ii and 4b,ii) and $36-48$ (Fig. 4a,iv and 4b,iv). For G3 and G3_NS this is for hours $36-60$ (Fig. 4c,iv-v and Fig. 4d,iv-v). We have avoided analysing hours $0-12$ in each case because the storm structure is still developing. Comparing KF and BMJ, we note the largest track difference between the hours of $12-24$ occurs during hours $12-18$, where the difference between the two tracks is $37^{\circ}$. For hours 36 -48 , the largest difference occurs during the 6 hours of $36-42$, where the difference is $32^{\circ}$. Comparing G3 and G3_NS, for hours 36 - 48, the largest difference occurs in the 6 hour period of hours $36-42$ and is $13^{\circ}$. For hours $48-60$, the greater difference occurs during hours $54-60\left(10^{\circ}\right)$. We analyse these important periods of difference and examine one of the time periods for KF / BMJ, and G3 / G3_NS more closely.

For Yasi, we identify several instances where there is significant disagreement between the simulated TC motion and the WN-1 PVT (Table 6, column 4). The differences between the simulated TC motion and WN-1 PVT for KF are large in every instance. In contrast, the only time $\mathrm{BMJ}$ showed moderate disagreement $\left(30^{\circ}\right)$ was during hours $18-24$. Returning to the key times of track difference between the schemes, during hours $12-18, \mathrm{KF}$ 
544 showed a PVT direction disagreement of $113^{\circ}$ and BMJ only $7^{\circ}$ (Table 6; compare Fig. 4a,ii 545 and 4b,ii). For hours $36-42$, KF showed a disagreement of $60^{\circ}$ and BMJ only $17^{\circ}$ (Table 6; 546 compare Fig. 4a,iv and 4b,iv). During both these periods, KF was not subjected to any 547 substantial steering, while BMJ was strongly steered. This is supported by the better WN-1 548 and TC motion agreement in BMJ. As previously stated, in the case of a westward moving storm in the Australian region like Yasi, beta drift adds an additional southward component of motion to the steering flow (Holland 1984; Carr and Elsberry 1990), which would explain the simulated motion vector of the storm shown in Fig. 4b,iv. KF, while partially subjected to the same influence, may be affected more by an evolving convective asymmetry, as the agreement between the TC motion and the diagnosed WN-1 PVT is not as good. This is therefore a possible cause for the track difference between KF and BMJ.

We can test this idea by examining the convection over one of the time periods where there is greater disagreement. For the second period of track deviation between the two cases, over hours $36-42$, we show a difference of $60^{\circ}$ between the WN-1 PVT and the TC motion for the KF run (Fig. 5). We also note the steering current magnitude in $\mathrm{KF}$ is $1.3 \mathrm{~m} \mathrm{~s}^{-1}$, and for BMJ is $4.4 \mathrm{~m} \mathrm{~s}^{-1}$. The direction of the steering in $\mathrm{KF}$ is $112^{\circ}$ but for BMJ $230^{\circ}$, only $3^{\circ}$ different from the TC motion $\left(227^{\circ}\right)$. The small disagreement between the WN-1 PVT and the $\mathrm{TC}$ motion for BMJ $\left(17^{\circ}\right)$ indicates a strongly steered storm. For KF, however, because the steering is weak and because the disagreement is large, we suggest that the convection is ultimately responsible for this poor agreement between the WN-1 PVT and the simulated TC motion and the subsequent difference in the two TC tracks. This is further supported by the TC motion vectors in Fig. 4a,iv and 4b,iv and the two tracks in Fig. 2a (BMJ is further southwest, which indicates a storm undergoing stronger beta drift). If we examine the simulated convection for KF over the 36 - 42 time period (Fig. 6), we note that convective asymmetry is present. The maximum convection is shown to rotate around the storm over 
time, from hour 36 (Fig. 6a) where there is a region in the south east and north west, to hour 38 (Fig. 6b) where the convection has rotated clockwise. At hour 40 (Fig. 6c) and 42 (Fig. $6 \mathrm{~d})$, there is a predominance of convection to the north and west of the storm centre. In theory, this should act to deflect the TC away from the motion $\left(190^{\circ}\right)$ diagnosed by the $\mathrm{WN}-1$ PVT (indicated by the solid arrow in Fig. 5), to the simulated motion of $250^{\circ}$ (dashed arrow in Fig. 5). This approximately relates to the clockwise rotation of the convection in KF, particularly over the latter periods of hours $36-42$. We hypothesise that the TC is deflected by the convection, further to the west away from its PVT-diagnosed course. This deflection is derived due to the fact a TC can propagate left or right of the vertical shear vector via advection of PV by the vortex circulation. Convective asymmetries develop from this interaction, often from vortex tilt and stretching. The convective asymmetry can then deflect the TC.

For the G3 and G3_NS cases, we note that there is greater disagreement between the storm motion and the WN-1 diagnostic for the G3_NS case (Table 6). For instance, during hours 36 - 42, the track direction difference between the G3 and G3_NS cases was only $13^{\circ}$ but the WN-1 PVT vs. TC motion disagreement was $14^{\circ}$ for G3 and $47^{\circ}$ for G3_NS. The steering current magnitude and direction in G3 were $5 \mathrm{~m} \mathrm{~s}^{-1}$ and $240^{\circ}$, only $2^{\circ}$ different from the TC motion $\left(238^{\circ}\right)$. G3_NS had a steering magnitude and direction of $4.8 \mathrm{~m} \mathrm{~s}^{-1}$ at $247^{\circ}$ (the TC motion was $251^{\circ}$ ). Likewise, over hours $54-60$, when the track direction difference was only $10^{\circ}$, the WN-1 PVT v TC motion disagreement was $19^{\circ}$ and $72^{\circ}$ for G3 and G3_NS respectively. Here, the steering for G3 was $5.7 \mathrm{~m} \mathrm{~s}^{-1}$ at $224^{\circ}$ (the TC motion was $237^{\circ}$ ), while in comparison, G3_NS was $5.1 \mathrm{~m} \mathrm{~s}^{-1}$ at $240^{\circ}$ (the TC motion was $247^{\circ}$ ). Both cases had similar steering current magnitudes, and the angular difference between the TC motion and the steering current direction was insignificant in each case. The larger disagreement between the WN-1 PVT and simulated TC motion in G3_NS might be the result of convective effects. 
If we examine the convective field for G3_NS over 36 - 42 (Fig. 7), we note the

595

596

597

598

599

600

601

602

603

604

605

606

607

608

609

610

611

612

613

614

615

616

617

618

influence of convection, which is a possible reason for the substantial disagreement between the WN-1 PVT diagnostic and the TC motion. The storm is highly asymmetric and the direction of the asymmetry is rapidly changing. The maximum convection is initially mostly located in the same general direction as the TC motion, with the bulk of convection in the western quadrant of the storm environment, rotating clockwise over time. Here we have another case where the TC motion appears not yet to have fully adjusted to developing asymmetries in the convective patterns, resulting in a large disagreement between the actual and PVT-diagnosed storm motions, as suggested by Chan et al. (2002).

In summary, for Yasi we have a clear and systematic response in the convection for the KF and G3_NS cases. These two cases had the largest disagreement between the WN-1 PVT and the simulated TC motion, accompanied by a deflection of the TC track and a subsequent westward moving storm. In contrast, for the BMJ and G3 cases, where there was less disagreement, these storms also showed stronger steering. This resulted in the TC track following the diagnosed motion from the WN-1 PVT more closely, but also meant the additional southward component in the TC motion, driven by beta drift, caused these tracks to move further southward and poleward. We hypothesise that this led to separation from the KF and G3_NS tracks.

\subsubsection{Rita}

Simulations of hurricane Rita also produced a number of outcomes, particularly during the latter stages of the life cycle. The BMJ+6 (dashed blue in Fig. 2d) and G3+6 (dashed green in Fig. 2d) simulations produced more northward curvature and, being in the Northern Hemisphere, more subsequent poleward motion than the KF+6 (dashed red in Fig. 2d) and G3+6_NS (dashed green in Fig. 2f) simulations. This is the same systematic behaviour that the Yasi simulations displayed. There was more sensitivity to initial conditions 
than the Yasi simulations showed, but for this storm the sensitivity of track to the choice of $\mathrm{CP}$ scheme was still greater than the sensitivity to the initial conditions. Note we have analysed the +6 simulations here as these gave superior track and intensity results (except for TD+6, but this case is not analysed here).

Key periods of difference in track direction include hours 24-36 (compare Fig. 8a,iii and 8 b,iii) and hours 36-48 (Fig. 8a,iv and 8b,iv) and afterwards. The resulting subsequent difference in tracks between $\mathrm{KF}+6$ and $\mathrm{BMJ}+6$ is largely due to the differences over these key periods ( $24-48$ hours). With regard to the steering, we note that $\mathrm{BMJ}+6$ is subjected to a stronger and more poleward steering current than KF+6 over the latter stages of the simulation (compare the magnitude of the blue coloured vectors in Fig. 8a and 8b), which sufficiently explains the poleward drift of BMJ+6.

A similar comparison can be made for the G3+6 and G3+6_NS simulations (Fig. 8c and 8d). As was the case in Yasi, the G3+6 and G3+6_NS TC tracks are not separated by as large a distance as seen in $\mathrm{KF}+6$ and $\mathrm{BMJ}+6$, but rather result from gradual separation occurring after the first 12 hours. Key periods of differences in track direction include hours 24-36 (compare Fig. 8c,iii and 8d,iii) and hours 60-72 (Fig. 8c,vi and 8d,vi). In addition, the G3+6 NS run does not appear to be as strongly steered as the G3+6 run.

In order to explain the difference in simulation outcome, we now explore the $\mathrm{WN}-1$ PVT diagnostic for the key periods of difference, for both cases. Similarly to the Yasi analysis, for the purposes of examining track sensitivity, we use a 6 hour WN-1 PVT (Table 7). We have not analysed G3+6 and G3+6_NS for hours $24-30,36-42$, and $66-72$ because the track difference for these times is not substantial. Unlike Yasi, there are no instances where the disagreement between the simulated TC motion and the diagnosed motion from the WN-1 PVT is large. This means there must be another cause for the difference in TC track between each case. This is because if an evolving convective 
asymmetry were the cause, then we would expect one case to have large disagreements in the simulated versus diagnosed motion, compared to the other case. For example, for hours $36-$ 42, where the track difference between $\mathrm{KF}+6$ and $\mathrm{BMJ}+6$ was $25^{\circ}$, the disagreement between the simulated and diagnosed TC motion was only $9^{\circ}$ for $\mathrm{KF}+6$ and $8^{\circ}$ for $\mathrm{BMJ}+6$, clearly indicating that the evolution of convective asymmetry is not the cause here. Where there is no disagreement, as here, then a component of the WN-1 PVT equation (e.g. steering current, beta drift or diabatic heating) must be responsible for the different simulated TC track outcome in this case. We examine the steering current for $36-42$ hours. If steering were responsible for the track difference between these two cases, then we should see some difference in the $\mathrm{KF}+6$ and $\mathrm{BMJ}+6$ steering. In $\mathrm{KF}+6$, the steering current direction is $265^{\circ}$ with a magnitude of $1.62 \mathrm{~m} \mathrm{~s}^{-1}$. For BMJ+6, the direction is $264^{\circ}$ at $1.6 \mathrm{~m} \mathrm{~s}^{-1}$. Both these values are similar to the $36-48$ hour value for each case (see Fig. 8 a,iv and 8b,iv). There is no significant difference in the steering between these two cases. Given this similarity, the difference in track may be due to differences in beta drift or diabatic heating. Here we note that the diabatic heating field, which incorporates stratiform heating, is a more complete representation of the possible effects on storm motion than convective heating alone (e.g. Wang and $\mathrm{Wu}$ 2004). The WN-1 diagnostic will include convective effects if they are correctly simulated, such that if the agreement between the TC motion and the WN-1 PVT is good, then the model is capturing the diabatic heating process well. If not, then changes in motion might be related to the diabatic heating field. This is particularly relevant if the steering and beta drift cannot explain the track difference between two cases. al. (2010, see their Fig. 1), in the case of $\mathrm{KF}+6$, a westward moving storm during this time $\left(279^{\circ}\right)$, the beta drift would be to the west $\left(270^{\circ}\right)$. The steering current vector would therefore 
need to be to the right of the TC motion vector here, but this is not the case (6 hour motion vectors not shown, but similar to that in Fig. 8a,iv). We show this concept in a schematic diagram (Fig. 9a). Fig. 9 shows schematically the contrasting relationship between TC motion, steering currents and the beta effect. In Fig. 9a, a storm travelling towards the northwest in the Northern Hemisphere would experience a beta drift towards the west. In contrast, Fig. 9b shows that a storm moving more to the west would experience a beta drift roughly in the same direction. Therefore, we conclude that the TC motion is not consistent with the combined steering and beta effect, as the beta effect alone does not explain the motion of $\mathrm{KF}+6$. For BMJ+6, a westward moving storm $\left(254^{\circ}\right)$, the beta effect is also to the west $\left(270^{\circ}\right)$. There is likely an effect in $\mathrm{BMJ}+6$ of convective processes as represented here by diabatic heating (Fig. 9b). This is because, unlike in Fig. 9a, where the beta effect alone does not explain the motion of $\mathrm{KF}+6$, in Fig. $9 \mathrm{~b}$ the beta effect is consistent with the steering current, so that other sources likely contribute to the deflection of the TC track. We examine the extent to which diabatic heating can explain the deflection of the track in the BMJ+6 case. The WN-1 diabatic heating at 42 hours shows quite contrasting fields for KF+6 (Fig. 10a) and BMJ+6 (Fig. 10b). The diabatic heating maximum for BMJ+6 is consistent with the TC motion for this time because the location of the maximum heating matches that of the simulated TC motion direction, but in $\mathrm{KF}+6$, the heating maximum is in the opposite direction to the motion. This indicates there are diabatic heating effects in $\mathrm{KF}+6$ that are contributing to the track difference in some way, but are not necessarily identifiable through diabatic heating asymmetry, as the simulated TC motion and the WN-1 PVT were in good agreement.

We find a similar result for hours $42-48$, where the track difference between $\mathrm{KF}+6$ and $\mathrm{BMJ}+6$ is $18^{\circ}$, but the $\mathrm{WN}-1$ PVT comparison to the $\mathrm{TC}$ motion indicates good agreement in both cases $\left(1^{\circ}\right.$ difference for $\mathrm{KF}+6,17^{\circ}$ for $\left.\mathrm{BMJ}+6\right)$. The steering current for 
694

695

696

697

698

699

700

701

702

703

704

705

706

707

708

709

710

711

712

713

714

715

716

717

718

this time period is $259^{\circ}$ at $1.4 \mathrm{~m} \mathrm{~s}^{-1}$ in $\mathrm{KF}+6$, compared to $255^{\circ}$ at $1.3 \mathrm{~m} \mathrm{~s}^{-1}$ in BMJ+6. The two are similar, indicating steering is not likely responsible for the track difference. In neither case is the motion consistent with the steering current direction as modified by the expected beta effect (not shown), implying that a difference in diabatic heating is one possible cause for the track difference.

With regard to the G3+6 and G3+6_NS cases, for hours 30 - 36, where the track difference was $17^{\circ}$, the $\mathrm{WN}-1$ PVT comparison to the TC motion yielded a disagreement of only $15^{\circ}$ for $\mathrm{G} 3+6$ and $14^{\circ}$ for G3+6_NS (Table 7), showing that the agreement is good. In both cases the TC is deflected slightly to the right of the WN-1 PVT diagnostic (not shown). The steering current for G3+6 at this time is $261^{\circ}$ at $2.2 \mathrm{~m} \mathrm{~s}^{-1}$, for G3+6_NS it is $254^{\circ}$ at 2.5 $\mathrm{m} \mathrm{s}^{-1}$. It is plausible that a steering direction difference of $7^{\circ}$ may be enough to explain the difference in the two TC tracks over this time, but this seems unlikely. For these westward moving storms, the direction of the beta drift would also be westward. In both cases, the motion is not consistent with the steering current direction as modified by the expected beta effect (not shown), so we have a similar situation to the KF and BMJ cases, where diabatic heating effects may explain the track difference. Rates of heating in G3+6 (Fig. 10c) are shown to be greater than G3+6_NS (Fig. 10d) for this time period. The location of the maximum is different also. In G3+6 this tends to oppose the direction of motion, and in G3+6_NS this is in the same direction of motion. G3+6 is tracking further to the north of G3+6_NS, possibly because the diabatic heating maximum in the G3+6 run is to the north.

In summary, Rita is a case where the agreement between the WN-1 PVT and the simulated TC motion was good. Unlike Yasi, where the time evolution of the convective effects in two of the CP schemes, as shown through poor agreement with the WN-1 PVT, was a possible cause of the track difference between cases, we do not find this for Rita. We showed that, in the case of $\mathrm{KF}+6 / \mathrm{BMJ}+6$, the track difference is either the result of beta drift 
or diabatic heating differences in BMJ+6 (hours $36-42$ ), or diabatic heating differences alone (hours $42-48$ ). In the case of G3+6/G3_NS+6, the track difference may be the result of diabatic heating differences over 30 - 36 hours and steering current differences over $60-$ 66 hours.

\subsubsection{Megi}

As the simulated tracks in Fig. 2 indicated, super typhoon Megi proved to have the most variation among simulations, that is, there was less consistent grouping between some simulations (e.g. KF and TD and G3_NS tracks together, and BMJ and G3 tracks together, as seen in Yasi and Rita). There was still a difference, however, between the G3 and G3_NS and the BMJ and KF cases. In fact, G3_NS and G3 (green coloured tracks in Fig. 2i) varied the most of any pairing across the three cyclone cases.

In the case of the TC motion for KF (Fig. 11a) and BMJ (Fig. 11b), differences in the motion in the early stages contribute to the difference in the tracks later. In addition, the looping of the BMJ track between hours $72-96$ is reflected in the TC motion vectors (Fig. $11 \mathrm{~b}$,vii-viii). During the first 12 hours, $\mathrm{KF}$ is tracking at $180^{\circ}$ and $\mathrm{BMJ}$ at $281^{\circ}$. The subsequent track difference over hours $12-36$ is an artefact of the initial displacement. Note that it is possible for a TC to move faster than the steering current, usually a sign of a dominant beta effect (e.g. Carr and Elsberry 1997). The period of 36 - 48 hours is a key period of difference between the cases: KF tracks at $297^{\circ}$ and $\mathrm{BMJ}$ at $317^{\circ}$, a $20^{\circ}$ difference. The following 12 hours $(48-60)$ proves to be another period of difference. KF tracks at $296^{\circ}$ and $\mathrm{BMJ}$ at $306^{\circ}$. Both cases show a rapid increase in TC speed, so that BMJ is now at a similar longitude to $\mathrm{KF}$ but due to the track difference, much further north (KF at $16.1{ }^{\circ} \mathrm{N}$, $133.7^{\circ} \mathrm{E}$; BMJ at $16.9^{\circ} \mathrm{N}, 134^{\circ} \mathrm{E}$ ). During the final stages prior to landfall, KF shifts course to $254^{\circ}$ over hours $96-108$, taking it further south of BMJ. KF continues to track similarly in this direction over hours 108 - 120 to make landfall further south of BMJ, which due to the 
96 - 108 motion has resulted in landfall much further north and some 12 hours after KF.

Like the Yasi and Rita cases, for Megi there was sensitivity to the use of shallow convection in the G3 scheme (Fig. 2i). G3 does not make landfall and tracks well to the north of G3_NS, which follows a course similar to the best track (black solid line in Fig 2i). The result for this parameterisation shows a systematic sensitivity to the shallow convection, as also observed in Yasi and Rita.

The key difference between the two cases occurs over hours 48 - 60 (Fig. 11c,v and $11 \mathrm{~d}, \mathrm{v})$ when G3 tracks at $284^{\circ}$ and G3_NS $295^{\circ}$, for a difference of $11^{\circ}$. At the end of 60 hours, this key difference in TC motion has G3_NS further north of G3. Over hours $72-84$ (compare Fig. 11c,vii and 11d,vii), G3 shows a northward curvature in its track. This is reflected in the steering vectors, where the storm is now more strongly steered over these latter times (Fig. 11c,vii-x), compared to G3_NS which is not nearly as strongly steered (Fig. 11d,vii-x).

G3_NS makes landfall sometime during the 108 - 120 period, but G3 makes a sudden turn to the north at hour 120 . This is illustrated by additional vector plots for hours $120-132$ and $132-144$ (Fig. 12). The steering current for $120-132$ hours is $53^{\circ}$ at $3.8 \mathrm{~m} \mathrm{~s}^{-1}$ and this increases in magnitude to $4.8 \mathrm{~m} \mathrm{~s}^{-1}$ with a direction of $54^{\circ}$ for hours $132-144$. Clearly G3 is being strongly steered over this time so that the storm continues to drift north. This result is most likely a function of the storm slowing from hour 60, relative to G3_NS, which increased in speed and translated the TC further west. This would have placed G3 in a different steering environment compared to G3_NS and this slowing of G3, possibly also driven by differences in simulated convection, has led to a different simulated outcome.

We test this hypothesis for both Megi convective comparisons. The results of the WN-1 PVT diagnostic for Megi are shown in Table 8. We analyse the WN-1 PVT for the time periods where the 6 hour TC track difference was large. We have once again calculated 
the WN-1 PVT as a 6 hour time tendency to minimise the effect of storm movement, so we analyse the key periods of track difference by splitting the 12 hour motion vector into two 6 hour periods. There are also some additional time periods analysed to explore some of the track behaviour noted later in the simulation.

We note that similarly to Rita, the overwhelming majority of the periods analysed show good agreement between the simulated TC motion and the WN-1 PVT diagnosed motion. There is only one case where the agreement is poor (BMJ). This indicates that time evolution of convective asymmetry is a possible cause for track deviation in the KF and BMJ comparison. For hours $42-48$, the difference between the WN-1 PVT and the TC motion in $\mathrm{KF}$ is only $9^{\circ}$, but for $\mathrm{BMJ}$ it is $42^{\circ}$ (Table 8 ). This is accompanied by the $28^{\circ}$ separation in these tracks as stated. For hours $48-54$, the disagreement between the WN-1 PVT and TC motion in $\mathrm{KF}$ is $13^{\circ}$ and for $\mathrm{BMJ}$ it is $29^{\circ}$. The diagnostic disagreement therefore decreased from the previous time to $16^{\circ}$, which may be indicative of a decrease in deflection of BMJ. To show that convective effects are a possible reason for the poor agreement in BMJ and subsequent track difference from KF, we show a time series of simulated reflectivity (Fig. 13). There is clearly a bias of convection to the north of the eye and the asymmetry is present across the entire 6 hour period. The TC is likely deflected toward the region of convective asymmetry. Here we see another possible example of an angular shift in the direction of maximum WN-1 PVT occurring before a corresponding change in direction of the storm motion, in this case possibly driven by transient convective effects. This causes the disagreement to decrease with time for the BMJ run.

For the track deviation in G3 and G3_NS, the results indicate the agreement between the WN-1 PVT and the simulated TC motion was good for both cases during the key times of track difference. We hypothesise that the simulated convection in both cases (not shown) is not anomalous enough to cause significant disagreement between the WN-1 PVT and the 
simulated TC motion, but nevertheless it is different between the two cases, so that a track difference between G3 and G3_NS arises. In order to assess the cause of the track difference, we undertake the same assessment that we performed for Rita. We note that because the convective effects are associated with the diabatic heating, that this component of the PVT diagnostic equation is most likely to be the cause for the track difference rather than the steering or beta effect. With regard to the steering current for hours $48-54$, for G3 this was $311^{\circ}$ at $2.4 \mathrm{~m} \mathrm{~s}^{-1}$ and $299^{\circ}$ at $2.4 \mathrm{~m} \mathrm{~s}^{-1}$ for G3_NS, indicating similar steering. An analysis of the beta effect shows that the TC motion is consistent with the steering current direction as modified by the expected beta effect in both cases (not shown). Therefore, in the absence of differences in either of those two components of the PVT diagnostic equation, a difference between the simulations in the diabatic heating is the most likely cause of the track difference. Indeed, we note there are contrasts in the diabatic heating of these storms during this time. The WN-1 diabatic heating at time 54 (Fig. 14) shows the location of the maximum rate of heating aligns with the track direction in each case. In G3 (Fig. 14a), the heating maximum closest to the storm center is southwest and in G3_NS (Fig. 14b) this is north. G3_NS is tracking further north of G3 possibly because its diabatic heating maximum is to the north, whereas G3 tracks further south of G3_NS because the diabatic heating maximum is to the south. This is driven by convective differences and indicates there are convective effects in both cases that are contributing to the track difference, but are not necessarily identifiable in the analysis conducted here.

In summary, Megi is another case where the majority of WN-1 PVT comparisons to the simulated TC track were in good agreement. The BMJ case, was the only case in poor agreement. The simulated track difference between BMJ and KF was largely the result of convective asymmetry in BMJ occurring over hours 42 - 54. For G3 and G3_NS, we showed that the simulated convection in both cases was not anomalous enough to cause significant 
disagreement between the WN-1 PVT and the simulated TC motion, but was nevertheless different between the two cases. This difference in convection was illustrated through the contrasting fields of the WN-1 diabatic heating for G3 and G3_NS.

\section{Summary and conclusions}

The objective of this study was to ascertain and attempt to quantify how sensitive tropical cyclone (TC) tracks are to changing the convective parameterization scheme in the WRF model. A diagnostic study of three major hurricanes from different basins was undertaken, using the Kain-Fritsch (KF), Betts-Miller-Janjic (BMJ), Grell-3D (G3) and Tiedtke (TD) convective parameterization (CP) schemes. In order to assess the track differences and explain the physical mechanisms of these differences, we used several diagnostics, including differences in steering currents and a $\mathrm{WN}-1$ potential vorticity tendency diagnostic for TC motion derived by Wu and Wang (2001) and Chan et al. (2002).

The major findings of this study are as follows:

1. The sensitivity of TC track to initial conditions (the initialisation time and model domain size) is less than the sensitivity of TC track to changing the convective parameterizations scheme.

2. The TC track is not particularly sensitive to the specification of shallow convection in the $\mathrm{KF}$, BMJ and TD schemes, but is highly sensitive in the G3 scheme. This is a systematic finding across all three cyclone cases.

3. The G3 simulation consistently performed poorly across all three cyclone cases. The no shallow simulation, G3_NS, was consistently better performing.

4. In general, agreement between the model-simulated track and the $\mathrm{WN}-1$ diagnostic is good, but with important exceptions that are often associated with model-generated 
convective asymmetries. Storms that are more strongly steered appear to show better agreement between the TC track and the $\mathrm{WN}-1$ diagnostic.

We have shown greater sensitivity to TC track from changing the $\mathrm{CP}$ scheme than from initial condition changes. The contribution of initial condition error to model forecast error is a topic of ongoing research, with sophisticated attempts to improve model initialisation for TC forecasting (e.g. ensemble Kalman filter, data assimilation etc. Kalnay 2003). Here we used WRF in its standard configuration with no additional initialisation techniques such as bogussing or data assimilation. We were able to reproduce both the TC track and intensity to a reasonable standard, with minimal TC track error. It is arguable whether there would be significant improvement from the use of more advanced initialisation techniques in these cases, but we might expect some improvement in simulated intensity and TC track. Running the same simulations again with a different initial dataset (e.g. ECMWF analyses) would be useful to assess the sensitivity to the forcing dataset, but it still seems unlikely the sensitivity to initial conditions would be larger than the change from CP. Moreover, the initialisation time chosen to test for initial condition sensitivity was only 6 hours later. While this should be adequate to test initial condition sensitivity, simulations from a different stage of TC life cycle (e.g. the intensification or mature stage) might show sensitivity to initial conditions. This would require a good assimilation / bogussing scheme and such tests are beyond the scope of this study, but recommended for future work.

Errors in TC track forecasts tend to be related to wind errors associated with the simulation of the large scale synoptic environment (Carr and Elsberry 2000). TC motion is controlled by the large scale synoptic flow (environmental wind) over the tropospheric deep layer, which is driven by the large scale weather systems in the vicinity combined with the beta effect (Holland 1983). Therefore it is important to capture the large scale synoptic environment to help minimise errors in TC motion. We addressed this issue by running a 
sufficiently large coarse domain (5076 $\times 3888 \mathrm{~km}$ of $12 \mathrm{~km}$ grid spacing) and by using the 1 $\times 1$ degree NCEP FNL data (i.e. a final analysis dataset comprising $10 \%$ more observations than GFS; Environmental Modeling Center, 2003). As a by-product of changing the model domain size, we were then able to assess the sensitivity to initialisation of the large scale environment and its effect on the TC track. Since the sensitivity in TC track from model domain size changes was less than that of changing the $\mathrm{CP}$ scheme, we can conclude there is greater sensitivity in TC motion to changing the $\mathrm{CP}$ scheme. This is likely a function of each CP representing the initial environment slightly differently, but also a result of the dominating effect of the $\mathrm{CP}$ scheme on TC structure through its representation of convection. scheme, and perhaps more importantly, the studies which have done so are varied in their methodological approach. Recent work (e.g. Sun et al. 2013; Sun et al. 2014a,b; Sun et al. 2015) has drawn conclusions about schemes specific to a particular storm event and has not attempted to reach systematic conclusions about $\mathrm{CP}$ performance. As an example of the varied nature of outcomes in this field, Sun et al. (2013) found that G3 presented better simulation results than $\mathrm{KF}$ and $\mathrm{BMJ}$ for storm intensity and structure. In the present study, we showed that G3 often produced the poorest representation of structure when compared against observations from each of the cases (e.g. an eye that was too large, an excessively large storm size). diagnostic was applied, is also to our knowledge the first instance where it has been applied to study TC track sensitivity to CP. Chan et al. (2002) states this method is useful for evaluating numerical weather prediction model performance, and whether the maximum cyclonic PVT can be captured by the model and whether this aligns with the TC motion. We showed that the WN1-PVT diagnostic is generally a good indicator of TC motion, but when 
892

893

894

895

896

897

898

899

900

901

902

903

904

905

906

907

908

909

910

we assessed the WN-1 PVT diagnosed motion against the simulated TC motion for each individual CP scheme, we showed some instances of disagreement. The Rita and Megi hurricane case studies generally showed good agreement between the WN-1 PVT and the simulated TC motion, where Yasi showed large differences for two of the CPs. the likely result of simulated convective asymmetry.

With regard to implications for modelling of TCs, we can make some general statements about what these findings mean from a modelling perspective. In order to make a general statement about $\mathrm{CP}$ performance, including which scheme is best at representing the large scale environment and convective processes in the TC vortex, many more cases would be required. What we have shown here, however, is that there is a systematic sensitivity of TC track to $\mathrm{CP}$ scheme that we established across three cases from different basins. In particular, the G3 scheme is highly sensitive to shallow convection, whereas the KF, TD and BMJ schemes are not. More accurate TC track and intensity representation was achieved with the no shallow convection configuration of G3. The WRF model successfully captures the cyclonic maximum of the WN-1 PVT and this diagnostic can be applied to successfully diagnose dynamical differences in TC motion as simulated by parameterisation schemes. This technique could also be applied to test sensitivity to other parameterisations (e.g. microphysics). 


\section{Acknowledgements}

912 This research was undertaken with the assistance of resources from the National 913 Computational Infrastructure (NCI), which is supported by the Australian Government. 914 Further funding was provided by the Edward and Isobel Kidson Scholarship through 915 Universities New Zealand and AMP New Zealand, and the Australian Postgraduate Award. 916 The authors would also like to thank Jimy Dudhia for scientific advice and Cindy Bruyère for 917 comments on an earlier draft.

918 


\section{References}

920 Arakawa A (2004) The Cumulus Parameterisation Problem: Past, present, and future.

921 J. Climate. 17:2493-2525.

922

923 Arakawa A, and Schubert WH (1974) Interaction of a cumulus cloud ensemble with the

924 large-scale environment, Part I. J. Atmos. Sci. 31:674-701.

925

926 Bassill NP (2014) Accuracy of early GFS and ECMWF Sandy (2012) track forecasts:

927 Evidence for a dependence on cumulus parameterization. Geophys. Res. Lett. 41:3274-3281.

928

929 Betts AK (1986) A new convective adjustment scheme. Part I: Observational and

930 theoretical basis. Quart. J. Roy. Meteor. Soc. 112:677-691.

931

932 Betts AK and Miller MJ (1986) A new convective adjustment scheme. Part II: Single column

933 tests using GATE wave, BOMEX, ATEX, and Arctic air-mass data sets. Quart. J. Roy. 934 Meteor. Soc. 112:693-709.

935

936 Bougeault P (1985) A simple parameterization of the large-scale effects of cumulus 937 convection. Mon. Wea. Rev. 113:2108-2121.

938

939 Bretherton CS, McCaa JR, and Grenier H (2004) A new parameterization for shallow 940 cumulus convection and its application to marine subtropical cloud-topped boundary layers.

941 Part I: Description and 1D results. Mon. Wea. Rev. 132:864-882.

942

943 Brisbane Times, cited 2015: Yasi bill revealed: \$800 million in damages. [Available online at 
http://www.brisbanetimes.com.au/queensland/yasi-bill-revealed-800-million-in-damages-

946

947 Cao Y, Fovell RG, and Corbosiero KL (2011) Tropical cyclone track and structure sensitivity 948 to initialization in idealised simulations: a preliminary study. Terr. Atmos. Ocean. Sci. $949 \quad 22: 559-578$.

950

951 Carr LE III, and Elsberry RL (1990) Observational evidence for prediction of tropical 952 cyclone propagation relative to environmental steering. J. Atmos. Sci. 47:542-46.

953

954 Carr, LE III, Elsberry R (1997) Models of tropical cyclone wind distribution and beta-effect 955 propagation for application to tropical cyclone track forecasting. Mon. Wea. Rev., 125:

956 $3190-3209$.

957

Cha D-H, Wang Y (2013) A dynamical initialization scheme for real-time forecasts of 959 tropical cyclones using the WRF model. Mon. Wea. Rev. 141:964-986.

960

961

Chan JCL, Ko FM, and Lei YM (2002) Relationship between potential vorticity tendency and 962 tropical cyclone motion. J. Atmos. Sci. 59:1317-1336.

963

964 Chan JCL, and Kepert JD (Eds.) (2010) Global Perspectives on Tropical Cyclones - From 965 Science to Mitigation. World Scientific Publishing, 444 pp.

966

967 Chan JC, and Williams RT (1987) Analytical and numerical studies of the beta-effect in 968 tropical cyclone motion. Part I: Zero mean flow. J. Atmos. Sci. 44:1257-1265. 
969 de Szoeke, SP, Wang Y, Xie S-P, and Miyama T (2006) The effect of shallow convection on 970 the eastern Pacific climate in a coupled model. Geophys. Res. Lett. 33, L17713. 971 doi:10.1029/2006GL026715.

972

973 Davis C, Wang W, Chen S, Chen Y, Corbosiero K, DeMaria M, Dudhia J, Holland G, Klemp 974 J, Michalakes J, Reeves H, Rotunno R, Snyder C, and Xiao Q (2008) Prediction of 975 Landfalling Hurricanes with the Advanced Hurricane WRF Model. Mon. Wea. 976 Rev. 136:1990-2005.

977

978 Dengler K, and Reeder MJ (1997) The effects of convection and baroclinicity on the 979 motion of tropical-cyclone-like vortices. Quart. J. Roy. Meteor. Soc. 123:699-725.

980

981

Dudhia J (1989) Numerical study of convection observed during the winter monsoon 982 experiment using a mesoscale two-dimensional model. J. Atmos. Sci. 46:3077-3107.

983

984 Emanuel KA (1989) The finite-amplitude nature of tropical cyclogenesis. J. Atmos. Sci. 985 $46: 3431-3456$

986

987

Emanuel KA (1991) A scheme for representing cumulus convection in large-scale models. J. 988 Atmos. Sci. 48:2313-2329.

989

990 Environmental Modelling Center (2003) The GFS atmospheric model. NCEP Office Note 991 442, Global Climate and Weather Modeling Branch, EMC, Camp Springs, Maryland.

992

993 Fovell RG, Corbosiero KL, and Kuo H-C (2009) Cloud microphysics impact on hurricane 
track as revealed in idealized experiments. J. Atmos. Sci. 66:1764-1778.

995

996 Frank WM (1983) The cumulus parameterisation problem. Mon. Wea. Rev. 111:1859-1871.

997

998 Frank WM, and Cohen C (1987) Simulation of tropical convective systems. Part I: A

999

cumulus parameterization. J. Atmos. Sci. 44:3787-3799.

1000

1001

Fritsch JM, and Chappell CF (1980) Numerical prediction of convectively driven

1002

mesoscale pressure systems. Part I: Convective parameterisation. J. Atmos. Sci. 37:172210031733.

1004

1005

Gregory D, and Rowntree PR (1990) A mass flux convection scheme with representation

1006

of cloud ensemble characteristics and stability-dependent closure. Mon. Wea. Rev.

1007

118:1483-1506.

1008

1009

Grell GA (1993) Prognostic evaluation of assumptions used by cumulus parameterisation.

1010

Mon. Wea. Rev. 121:764-787.

1011

1012 Grell GA, and Devenyi D (2002) A generalised approach to parameterising convection 1013 combining ensemble and data assimilation techniques. Geophys. Res. Lett., 29 1014 doi:10.1029/2002GL015311.

1015

1016 Han J, and Pan H-L (2011) Revision of convection and vertical diffusion schemes in the

1017 NCEP global forecast system. Wea. Forecasting. 26:520-533.

1018 
1019

1020

1021

1022

1023

1024

1025

1026

1027

1028

1029

1030

1031

1032

1033

1034

1035

1036

1037

1038

1039

1040

1041

1042 Kain JS (2004) The Kain-Fritsch convective parameterization: An update. J. Appl. Meteor. $1043 \quad 43: 170-181$.

Hill KA, and Lackmann GM (2009) Analysis of idealized tropical cyclone simulations using the Weather Research and Forecasting Model: Sensitivity to turbulence parameterization and grid spacing. Mon. Wea. Rev. 137:745-765.

Holland GJ (1983) Tropical cyclone motion: Environmental interaction plus a beta effect. J. Atmos. Sci. 40:328-342.

Holland GJ (1984) Tropical cyclone motion: a comparison of theory and observation. J. Atmos. Sci. 41:68-75

Janjic ZI (1990) The step-mountain coordinate: physical package. Mon. Wea. Rev. 118:1429-1443.

Janjic ZI (1994) The step-mountain coordinate model: Further developments of the convection, viscous sub-layer, and turbulence closure schemes. Mon. Wea.

Rev. 122:927-945.

Janjic ZI (1996) The surface layer in the NCEP Eta Model. 11th Conf. on Numerical Weather Prediction, Norfolk, VA, 19-23 August; Amer. Meteor. Soc., Boston, MA, 354-355.

Janjic ZI (2001) Nonsingular Implementation of the Mellor-Yamada Level 2.5 Scheme in the NCEP Mesoscale model. NCEP Office Note, No. 437, 61 pp. $43: 170-181$ 
1044 Kain JS, and Fritsch JM (1990) A one-dimensional entraining/detraining plume model and its 1045 application in convective parameterization. J. Atmos. Sci. 47:2784-2802.

1046

1047 Kain JS, and Fritsch JM (1993) Convective parameterisation for mesoscale models: The 1048 Kain-Fritsch scheme. The Representation of Cumulus Convection in Numerical Models, 1049 Meteor. Monogr., No. 46, Amer. Meteor. Soc., 165-170.

1050

1051

Kalnay E (2003) Atmospheric modeling, data assimilation, and predictability, Cambridge 1052 University Press, Cambridge, 341pp.

1053

1054 Kieu CQ, Truong NM, Mai HT, and Ngo-Duc T (2012) Sensitivity of the track and intensity 1055 forecasts of typhoon Megi (2010) to satellite-derived atmospheric motion vectors with the 1056 ensemble kalman filter. J. Atmos. Oceanic Technol. 29:1794-1810.

1057

1058

Knabb RD, Brown DP, and Rhome JR (2006) Hurricane Rita tropical cyclone report. 1059

National Hurricane Centre Report. Available at: http://www.nhc.noaa.gov/pdf/TCR1060 AL182005_Rita.pdf

1061

Krishnamurti TN, Han W, and Oosterhof D (1998) Sensitivity of hurricane intensity forecasts 1063 to physical initialization. Met. Atmos. Phys. 65:171-181.

1064

1065

Kumar A, Done J, and Dudhia J (2011) Simulations of cyclone Sidr in the Bay of Bengal 1066 with a high-resolution model: sensitivity to large-scale boundary forcing. Meteor. Atmos. Phys. 114:123-137. 
1069 Kuo HL (1974) Further studies of the parameterization of the influence of cumulus 1070 convection on large-scale flow. J. Atmos. Sci. 31:1232-1240.

1071

1072 Li X, and Pu Z (2009) Sensitivity of numerical simulations of the early rapid intensification 1073 of hurricane Emily to cumulus parameterization schemes in different model horizontal 1074 resolutions. J. Meteor. Soc. Japan. 87:403-421.

1075

1076

Mellor GL, and Yamada T (1982) Development of a turbulence closure model for 1077 geophysical fluid problems. Rev. Geophys. Sp. Phys. 20:851-875.

1078

1079 Mlawer EJ, Taubman SJ, Brown PD, Iacono MJ, and Clough SA (1997) Radiative transfer 1080 for inhomogeneous atmosphere: RRTM, a validated correlated-k model for the longwave. J. 1081 Geophys. Res. 102 (D14):16663-16682.

1082

1083

Molinari J, and Dudek M (1992) The parameterisation of convection in numerical models:

1084 A critical review. Mon. Wea. Rev. 120:326-344.

1085

1086

Nasrollahi N, AghaKouchak A, Li J, Gao X, Hsu K, and Sorooshian S (2012) Assessing the 1087 impacts of different WRF precipitation physics in hurricane simulations. Wea. Forecasting. $1088 \quad 27: 1003-1016$.

1089

1090

National Centers for Environmental Prediction/National Weather Service/NOAA/U.S. 1091 Department of Commerce (2000) NCEP FNL Operational Model global tropospheric 1092 analyses, continuing from July 1999. Research Data Archive at the National Center for 1093 Atmospheric Research, Computational and Information Systems Laboratory, Boulder, CO. 
[Available online at http://dx.doi.org/10.5065/D6M043C6.].

1095

1096

National Disaster Risk Reduction and Management Council, 2010: SitRep No. 12 Effects of

1097 Typhoon Megi. [Available online at:

1098 http://reliefweb.int/sites/reliefweb.int/files/resources/3B64B52B1B6A5457492577C3001AC

1099 81B-Full_Report.pdf].

1100

1101

Ooyama KV (1971) A theory on parameterization of cumulus convection. J. Meteor. Soc. 1102 Japan. 49:744-756.

1103

1104

1105 tropical cyclone recurvature. Mon. Wea. Rev. 132:1143-1166.

1106

1107

Prater BE, and Evans JL (2002) Sensitivity of modelled tropical cyclone track and 1108 structure of hurricane Irene (1999) to the convective parameterisation scheme. Meteor. Atmos. Phys. 80:103-115.

1110

1111

Reuters, cited 2015: Cyclone Yasi to cost insurers AUS \$3.5 bln - forecaster. [Available 1112 online at http://www.reuters.com/article/2011/02/03/insured-losses-yasi-idUSLDE7121NR20110203]

Rogers E, Black T, Ferrier B, Lin Y, Parrish D, and DiMego G (2001) Changes to the NCEP 1116 Meso Eta Analysis and Forecast System: Increase in resolution, new cloud microphysics, 1117 modified precipitation assimilation, modified 3DVAR analysis. NWS Technical Procedures 1118 Bulletin 488, NOAA/NWS. 
Rosier MJ, and Miller DK (2005) Sensitivity of Hurricane Charley simulations to changes in WRF model. Extended abstract, 21st Conf. on Weather Analysis and Forecasting/17th Conf. on Numerical Weather Prediction, Washington, DC, Amer. Meteor. Soc., P1.33. [Available online at: https://ams.confex.com/ams/WAFNWP34BC/techprogram/paper_94821.htm]

Skamarock, WC, Wang W, Barker D, Gill D, Klemp J, and Powers GJ (2008) A description of the Advanced Research WRF Version 3.1. NCAR Technical Note468+STR.

Srinivas CV, Venkatesan R, Bhaskar Rao DV, and Prasad DH (2007) Numerical Simulation of Andhra Severe Cyclone (2003): Model Sensitivity to the Boundary Layer and Convection Parameterization. Pure Appl. Geophys. 164:1465-1487.

Stensrud DJ (2007) Parameterisation schemes: Keys to understanding numerical models, Cambridge University Press, Cambridge, 459pp.

Sun Y, Lan Y, Zhong Z, Hu Y, and Ha Y (2013) Dependence of model convergence on horizontal resolution and convective parameterisation in simulations of a tropical cyclone at greyzone resolutions. J. Geophys. Res. 118:7715-7732.

Sun Y, Zhong Z, Lu W, and Hu Y (2014a) Why are tropical cyclone tracks over the western North Pacific sensitive to the cumulus parameterisation scheme in regional climate modelling? A case study for Megi (2010). Mon. Wea. Rev. 142:1240-1249.

\footnotetext{
Sun Y, Yi L, Zhong Z, and Ha Y (2014b) Performance of a new convective parameterisation
} 
scheme on model convergence in simulations of a tropical cyclone at grey-zone resolutions. J. Atmos. Sci. 71:2078-2088.

1146

1147 Sun Y, Zhong Z, Dong H, Shi J, and Hu Y (2015) Sensitivity of tropical cyclone track 1148 simulation over the Western North Pacific to different heating/drying rates in the BettsMiller-Janjic Scheme. Mon. Wea. Rev. doi:10.1175/MWR-D-14-00340.1, in press.

1150

1151

Tiedtke M (1989) A comprehensive mass flux scheme for cumulus parameterization in 1152 large-scale models. Mon. Wea. Rev. 117:1779-1800.

1153

1154

1155 track forecasts. Mon. Wea. Rev. 140:2188-2197.

1156 experiment. Mon. Wea. Rev. 132:274-296.

1160

1161

Wang Y, Xu H, and Xie S-P (2004b) Regional model simulations of marine boundary layer clouds over the southeast Pacific off South America. Part II: Sensitivity experiments. Mon. 1163 Wea. Rev. 132:2650-2668.

1164

1165

Wang, Y, and Wu CC (2004). Current understanding of tropical cyclone structure and

1166 intensity changes-a review. Met. Atmos. Phys. 87: 257-278.

1168 Weisman ML, Skamarock WC, and Klemp JB (1997) The resolution dependence of 
explicitly modeled convective systems. Mon. Wea. Rev. 125:527-548.

1170

Wu CC, and Emanuel KA (1993) Interaction of a baroclinic vortex with background shear: Application to hurricane movement. J. Atmos. Sci. 50:62-76.

1173

1174

Wu CC, and Wang B (2000) A potential vorticity tendency diagnostic approach for tropical 1175 cyclone motion. Mon. Wea. Rev. 128:1899-1911.

1176

Wu CC, and Wang B (2001) Effects of convective heating on movement and vertical 1178 coupling of tropical cyclones: A numerical study. J. Atmos. Sci. 58:3639-3649.

1179

1180

Zhang C, Wang Y, and Hamilton K (2011) Improved representation of boundary layer clouds 1181 over the Southeast Pacific in ARW-WRF using a modified Tiedtke cumulus parameterization scheme. Mon. Wea. Rev. 139:3489-3513. dimensional tropical cyclone model. J. Atmos. Sci. 59:1825-1840.

1186

Zhu H, Smith RK, and Ulrich W (2001) A minimal three-dimensional tropical cyclone 1188 model. J. Atmos. Sci. 58:1924-1944. 
1194

1195

1196

1197

1198

1199

1200

1201

1202

1203

1204

1205

1206

1207

1208

1209

1210

1211

1212

1213

1214

1215

1216

1217

1218

Table 1 Summary of CP schemes used in this study

Table 2 Yasi landfall distance differences (in $\mathrm{km}$ ) for each $\mathrm{CP}$ (NS refers to NO_SHALLOW)

Table 3 Rita landfall distance differences for each CP

Table 4 Megi landfall distance differences for each CP. NB: * indicates the G3/G3+6 cases did not make landfall. In this instance we compare the position at the time of the first storm making landfall $(\mathrm{KF})$

Table 5 Comparison of the landfall of each simulation to the observed landfall for each of the cyclone cases. ${ }^{*}$ These Rita cases were ' +6 '. N/A indicates no landfall. $\dagger$ Indicates the average is only based on two cases due to a simulation not making landfall in one of the cases.

Table 6 Matrix of simulated TC motion (TC motion from the vector plots) and the diagnosed motion derived from the WN-1 PVT plots, for the Yasi case

Table 7 The same as Table 6, for the Rita case

Table 8 The same as Table 6, for the Megi case 


\section{List of Figures}

Fig. 1 The domain configurations for the (a) Yasi, (b) Rita, and (c) Megi cases. Domains 2 and 3 are moving nests. The resolution is 12,4 and $1.33 \mathrm{~km}$ respectively for each domain. The number of grid points were 423 × 324, $201 \times 201,240 \times 240$ for each domain Fig. 2 Tracks of the CP simulations for each case study for (a) Yasi 00Z (solid) and $+06 \mathrm{Z}$ (dashed), (b) Yasi 00Z (solid) and DOM2 (dashed), (c) Yasi 00Z (solid) and NS (dashed), (d) - (f) Rita, and (g) - (i) Megi. The observed track is the black line. The black dots represent the track position at 12 hourly intervals. The KF cases are in red, the BMJ cases are in blue, the G3 cases in green and TD in yellow

Fig. 3 Comparison of the trend in simulated pressure $(\mathrm{hPa})$ and six hour moving average of wind speed (knots), for $(\mathrm{a}-\mathrm{d})$ Yasi, $(\mathrm{e}-\mathrm{h})$ Rita, and (i - l) Megi. Panel (a) pressure, and (b) wind, compare the Yasi 00Z and +06Z simulations, and panel (c) and (d) the Yasi 00Z and NS simulations. For Rita, $(\mathrm{e}-\mathrm{f})$ 00Z and +06Z simulations, and $(\mathrm{g}-\mathrm{h})+06 \mathrm{Z}$ and +6_NS simulations. For Megi, $(\mathrm{i}-\mathrm{j})$ 00Z and +06Z simulations, and $(\mathrm{k}-\mathrm{l})$ 00Z and NS simulations. The ibTracs observed data is given by the black dots

Fig. 4 TC motion and steering current vectors (in $\mathrm{m} \mathrm{s}^{-1}$ ) for the Yasi simulation, for (a) KF, (b) BMJ, (c) G3, and (d) G3_NS. Each panel shows the vectors at 12-hr intervals, for (i) 0 12 hours, (ii) $12-24$ hours, (iii) $24-36$ hours, (iv) $36-48$ hours, (v) $48-60$ hours, (vi) 60 -72 hours, and (vii) $72-84$ hours

Fig. 5 WN-1 PV tendency (in PVU) for KF over simulation hours 36 - 42, for the Yasi case. The solid arrow indicates the diagnosed motion from the WN-1 PV tendency, while the dashed line is the simulated TC motion. Note: this is a southern hemisphere case, and as such blue indicates the cyclonic PV. The output is from domain 1 with a grid spacing of $12 \mathrm{~km}$, but has been coordinate transformed to a new $300 \times 300$ grid to account for storm movement over 6 hours. 1 PVU $=1.0 \times 10^{-6} \mathrm{~m}^{2} \mathrm{~s}^{-1} \mathrm{~K} \mathrm{~kg}^{-1}$ 
Fig. 6 Simulated radar reflectivity of Yasi for the KF case at (a) 06Z 01 February 2011 at 36 hours of simulation, (b) 08Z at 38 hours, (c) $10 \mathrm{Z}$ at 40 hours, and (d) $12 \mathrm{Z}$ at 42 hours

Fig. 7 The same as Fig. 6 for the G3_NS case at (a) 06Z 01 February 2011 at 36 hours, (b) 08Z at 38 hours, (c) $10 \mathrm{Z}$ at 40 hours, and (d) $12 \mathrm{Z}$ at 42 hours

Fig. 8 TC motion and steering current vectors (in $\mathrm{m} \mathrm{s}^{-1}$ ) for the Rita simulation, for (a) $\mathrm{KF}+6$,

(b) BMJ+6, (c) G3+6, and (d) G3+6_NS. Each panel shows the vectors at 12-hr intervals for,

(i) $0-12$ hours, (ii) $12-24$ hours, (iii) $24-36$ hours, (iv) $36-48$ hours, (v) $48-60$ hours,

1252

(vi) 60 - 72 hours, (vii) $72-84$ hours, (viii) $84-96$ hours, (ix) $96-108$ hours, and (x) $108-$ 120 hours (except for $\mathrm{KF}+6$ )

Fig. 9 Schematic diagram showing the TC motion (red line labelled 'TC'), actual steering 1255 (solid blue labelled 'S') and beta effect (solid black line labelled 'B') for (a) KF+6 $36-42$, 1256 and (b) BMJ+6 $36-42$. In (a), for the tropical cyclone track to be consistent with the steering and the beta effect, the steering current would have to be in the direction of the blue dashed line, with the black dashed line then being in the same direction as the beta effect. In (b), the TC motion is consistent with the steering and the beta effect as the steering is in the same direction as the beta effect. See the text for an explanation

Fig. $10 \mathrm{WN}-1$ diabatic heating $\left(\mathrm{Pa} \mathrm{K} \mathrm{s}{ }^{-1}\right.$ ) for Rita for (a) KF+6 at 42 hours, (b) BMJ+6 at 42

1262 hours, (c) G3+6 at 36 hours, and (d) G3+6_NS at 36 hours

1263 Fig. 11 TC motion and steering current vectors (in $\mathrm{m} \mathrm{s}^{-1}$ ) for the Megi simulation, for (a) KF, 1264 (b) BMJ, (c) G3, and (d) G3_NS. Each panel shows the vectors at 12-hr intervals, for (i) 0 1265 12 hours, (ii) $12-24$ hours, (iii) $24-36$ hours, (iv) $36-48$ hours, (v) $48-60$ hours, (vi) 60 1266 - 72 hours, (vii) $72-84$ hours, (viii) $84-96$ hours, (ix) $96-108$ hours, and (x) $108-120$ 1267 hours

1268 Fig. 12 TC motion and steering current vectors (in $\mathrm{m} \mathrm{s}^{-1}$ ) for 12-hr intervals for the Megi G3 1269 simulation, (a) $120-132$ hours, and (b) $132-144$ hours 
1270 Fig. 13 Simulated radar reflectivity of Megi for the BMJ case at (a) 06Z 15 at 42 hours, (b)

$127108 \mathrm{Z}$ at 44 hours, (c) $10 \mathrm{Z}$ at 46 hours, and (d) $12 \mathrm{Z}$ at 48 hours

1272 Fig. 14 The same as Fig. 10 for Megi at 54 hours for (a) G3, and (b) G3_NS 
Table 1 Summary of CP schemes used in this study

\begin{tabular}{|c|c|c|c|c|c|}
\hline Scheme & $\begin{array}{l}\text { Type of } \\
\text { scheme }\end{array}$ & $\begin{array}{l}\text { Cloud } \\
\text { detrainment }\end{array}$ & Closure & Comments & Shallow convection design \\
\hline BMJ & $\begin{array}{l}\text { Convective } \\
\text { Adjustment }\end{array}$ & $\mathrm{N}$ & $\begin{array}{l}\text { Sounding } \\
\text { adjustment }\end{array}$ & $\begin{array}{l}\text { Based on pre-defined } \\
\text { reference profiles. Deep } \\
\text { convection profiles depend on } \\
\text { cloud efficiency. }\end{array}$ & $\begin{array}{l}\text { Shallow convection is a process operating between a } \\
\text { buoyant layer and an inversion aloft. The shallow } \\
\text { scheme is used if cloud depth }<200 \mathrm{hPa} \text {, or if the } \\
\text { precipitation rate is negative. The cloud base is the } \\
\text { same as for the deep scheme, but the cloud top is } \\
\text { defined as the layer that has the fastest decrease in } \\
\mathrm{RH} \text {. }\end{array}$ \\
\hline
\end{tabular}




\begin{tabular}{|c|c|c|c|c|c|}
\hline Scheme & $\begin{array}{l}\text { Type of } \\
\text { scheme }\end{array}$ & $\begin{array}{l}\text { Cloud } \\
\text { detrainment }\end{array}$ & Closure & Comments & Shallow convection design \\
\hline G3 & $\begin{array}{l}\text { Ensemble } \\
\text { scheme } \\
\text { (Mass-flux } \\
\text { approach) }\end{array}$ & $\bar{Y}$ & $\begin{array}{l}\text { Various e.g. } \\
\text { CAPE, } \\
\text { moisture } \\
\text { convergence. }\end{array}$ & $\begin{array}{l}\text { Based on Grell- Devenyi } \\
\text { (Grell and Devenyi 2002) } \\
\text { scheme. Allows subsidence } \\
\text { spreading over neighbouring } \\
\text { grid cells. }\end{array}$ & $\begin{array}{l}\text { The fractional area coverage of convective updrafts is } \\
\text { first defined. This fractional area is related to the } \\
\text { entrainment rate. The entrainment rate determines } \\
\text { when the fractional area coverage of convection } \\
\text { becomes important. The maximum allowable } \\
\text { fractional coverage determines when the CP } \\
\text { transforms itself to a shallow convection scheme. }\end{array}$ \\
\hline
\end{tabular}


1274 Table 2 Yasi landfall distance differences (in $\mathrm{km}$ ) for each CP (NS refers to 1275 NO_SHALLOW)

\begin{tabular}{|c|c|c|c|c|c|c|c|}
\hline Case & $\begin{array}{l}v s . \\
+6\end{array}$ & $\begin{array}{c}v s . \\
D O M 2\end{array}$ & $\begin{array}{c}K F \text { vs. } \\
\text { other } \\
C P s\end{array}$ & $\begin{array}{l}v s . \\
N S \\
C P\end{array}$ & $\begin{array}{c}K F N S \\
\text { vs. other } \\
N S C P S\end{array}$ & $\begin{array}{c}\text { KF_DOM2 } \\
\text { vs. other } \\
\text { DOM2 } \\
\text { CPs }\end{array}$ & $\begin{array}{c}K F+6 \text { vs. } \\
\text { other }+6 \\
C P s\end{array}$ \\
\hline $\mathrm{KF}$ & 22 & 73 & - & 15 & - & - & - \\
\hline BMJ & 119 & 74 & 329 & 14 & 300 & 184 & 243 \\
\hline G3 & 86 & 61 & 280 & 278 & 21 & 181 & 229 \\
\hline TD & 10 & 15 & 100 & 37 & 84 & 153 & 84 \\
\hline AVERAGE & 59 & 55 & 236 & 86 & 135 & 172 & 185 \\
\hline
\end{tabular}

1276

1277

1278 Table 3 Rita landfall distance differences for each CP

\begin{tabular}{|c|c|c|c|c|c|c|c|}
\hline Case & $\begin{array}{c}v s . \\
O O Z\end{array}$ & $\begin{array}{c}\text { vs. } \\
\text { DOM2 }\end{array}$ & $\begin{array}{c}\text { KF+6 } \\
\text { vs. } \\
\text { other } \\
+6 \\
\text { CPs }\end{array}$ & $\begin{array}{c}v s . \\
+6 N S \\
C P\end{array}$ & $\begin{array}{c}\text { KF+6 } \\
N S \text { vs. } \\
\text { other } \\
+6 N S \\
\text { CPs }\end{array}$ & $\begin{array}{c}K+6 \_D O M 2 \\
\text { vs. other } \\
+6 \_D O M 2 \\
\text { CPs }\end{array}$ & $\begin{array}{c}\text { KF vs. } \\
\text { other } \\
\text { ooZ CPs }\end{array}$ \\
\hline $\mathrm{KF}+6$ & 29 & 97 & - & 41 & - & - & - \\
\hline $\mathrm{BMJ}+6$ & 202 & 73 & 350 & 57 & 429 & 320 & 562 \\
\hline $\mathrm{G} 3+6$ & 125 & 144 & 32 & 287 & 74 & 359 & 464 \\
\hline TD+6 & 143 & 45 & 40 & 65 & 78 & 96 & 126 \\
\hline AVERAGE & 124 & 89 & 237 & 112 & 193 & 258 & 384 \\
\hline
\end{tabular}


1283 Table 4 Megi landfall distance differences for each CP. NB: * indicates the G3/G3+6 cases 1284 did not make landfall. In this instance we compare the position at the time of the first storm 1285 making landfall (KF)

\begin{tabular}{|c|c|c|c|c|c|c|c|}
\hline Case & $\begin{array}{l}v s . \\
+6\end{array}$ & $\begin{array}{c}v s . \\
\text { DOM2 }\end{array}$ & $\begin{array}{c}\text { KF vs. } \\
\text { other } \\
C P s\end{array}$ & $\begin{array}{l}v s . \\
N S \\
C P\end{array}$ & $\begin{array}{c}\text { KF NS } \\
\text { vs. other } \\
N S C P s\end{array}$ & $\begin{array}{c}K F_{-} \text {DOM2 } \\
\text { vs. other } \\
\text { DOM2 } \\
\text { CPs }\end{array}$ & $\begin{array}{c}\text { KF+6 vs. } \\
\text { other }+6 \\
C P s\end{array}$ \\
\hline $\mathrm{KF}$ & 53 & 74 & - & 10 & - & - & - \\
\hline BMJ & 45 & 73 & 183 & 2 & 189 & 200 & 188 \\
\hline G3 & $249 *$ & $248 *$ & $581 *$ & 343 & 289 & $793 *$ & $718^{*}$ \\
\hline TD & 34 & 113 & 251 & 100 & 160 & 212 & 260 \\
\hline AVERAGE & 95 & 127 & 338 & 113 & 212 & 401 & 388 \\
\hline
\end{tabular}

1286

1287

1288

1289

1290

1291

1292

1293

1294

1295

1296

1297

1298

1299

1300

1301

1302

1303

1304 
1305 Table 5 Comparison of the landfall of each simulation to the observed landfall for each of the 1306 cyclone cases. *These Rita cases were ' +6 '. N/A indicates no landfall. $†$ Indicates the average 1307 is only based on two cases due to a simulation not making landfall in one of the cases

\begin{tabular}{|c|c|c|c|c|}
\hline Case & $\begin{array}{c}\text { Yasi vs. observed } \\
\text { landfall }(\mathrm{km})\end{array}$ & $\begin{array}{c}\text { Rita vs. } \\
\text { observed } \\
\text { landfall }(\mathrm{km})\end{array}$ & $\begin{array}{l}\text { Megi vs. observed } \\
\text { landfall }(\mathrm{km})\end{array}$ & $\begin{array}{l}\text { Average (from } \\
\text { all three cases) }\end{array}$ \\
\hline $\mathrm{KF}$ & 173 & 291 & 262 & 242 \\
\hline BMJ & 490 & 301 & 78 & 289 \\
\hline G3 & 445 & 192 & N/A & $318^{\dagger}$ \\
\hline TD & 80 & 165 & 14 & 86 \\
\hline $\mathrm{KF}+6$ & 158 & 268 & 306 & 244 \\
\hline $\mathrm{BMJ}+6$ & 373 & 98 & 122 & 197 \\
\hline $\mathrm{G} 3+6$ & 364 & 66 & N/A & $215 \dagger$ \\
\hline $\mathrm{TD}+6$ & 75 & 307 & 46 & 142 \\
\hline KF_NS & 188 & $301 *$ & 270 & 253 \\
\hline BMJ_NS & 475 & $153^{*}$ & 81 & 236 \\
\hline G3_NS & 170 & $236^{*}$ & 26 & 144 \\
\hline TD_NS & 117 & $373 *$ & 110 & 200 \\
\hline KF_DOM2 & 233 & $170^{*}$ & 328 & 243 \\
\hline BMJ_DOM2 & 415 & $154 *$ & 145 & 244 \\
\hline G3_DOM2 & 408 & $211 *$ & N/A & $309 \dagger$ \\
\hline TD_DOM2 & 89 & $266^{*}$ & 122 & 159 \\
\hline
\end{tabular}


1316 Table 6 Matrix of simulated TC motion (TC motion from the vector plots) and the diagnosed 1317 motion derived from the WN-1 PVT plots, for the Yasi case

\begin{tabular}{|c|c|c|c|}
\hline $\begin{array}{c}\text { Scheme and analysis } \\
\text { period }\end{array}$ & $\begin{array}{c}\text { Simulated direction } \\
\text { (TC motion) }\end{array}$ & $\begin{array}{c}\text { Diagnosed direction } \\
(W N-1 \text { PVT) }\end{array}$ & Disagreement \\
\hline $\mathrm{KF}(12-18)$ & $260^{\circ}$ & $147^{\circ}$ & $113^{\circ}$ \\
\hline BMJ (12 - 18) & $223^{\circ}$ & $230^{\circ}$ & $7^{\circ}$ \\
\hline $\mathrm{KF}(18-24)$ & $255^{\circ}$ & $196^{\circ}$ & $59^{\circ}$ \\
\hline BMJ (18 - 24) & $246^{\circ}$ & $216^{\circ}$ & $30^{\circ}$ \\
\hline $\mathrm{KF}(36-42)$ & $250^{\circ}$ & $190^{\circ}$ & $60^{\circ}$ \\
\hline $\mathrm{BMJ}(36-42)$ & $218^{\circ}$ & $235^{\circ}$ & $17^{\circ}$ \\
\hline $\mathrm{KF}(42-48)$ & $245^{\circ}$ & $210^{\circ}$ & $35^{\circ}$ \\
\hline $\mathrm{BMJ}(42-48)$ & $236^{\circ}$ & $242^{\circ}$ & $6^{\circ}$ \\
\hline G3 $(36-42)$ & $238^{\circ}$ & $224^{\circ}$ & $14^{\circ}$ \\
\hline G3_NS $(36-42)$ & $251^{\circ}$ & $204^{\circ}$ & $47^{\circ}$ \\
\hline G3 $(42-48)$ & $234^{\circ}$ & $225^{\circ}$ & $9^{\circ}$ \\
\hline G3_NS $(42-48)$ & $245^{\circ}$ & $205^{\circ}$ & $40^{\circ}$ \\
\hline G3 $(48-54)$ & $228^{\circ}$ & $227^{\circ}$ & $1^{\circ}$ \\
\hline G3_NS $(48-54)$ & $237^{\circ}$ & $190^{\circ}$ & $47^{\circ}$ \\
\hline G3 $(54-60)$ & $237^{\circ}$ & $218^{\circ}$ & $19^{\circ}$ \\
\hline G3_NS $(54-60)$ & $247^{\circ}$ & $175^{\circ}$ & $72^{\circ}$ \\
\hline
\end{tabular}


Table 7 The same as Table 6, for the Rita case

\begin{tabular}{|c|c|c|c|}
\hline $\begin{array}{c}\text { Scheme and analysis } \\
\text { period }\end{array}$ & $\begin{array}{l}\text { Simulated direction } \\
\text { (TC motion) }\end{array}$ & $\begin{array}{c}\text { Diagnosed direction } \\
\quad(W N-1 \text { PVT) }\end{array}$ & Disagreement \\
\hline $\mathrm{KF}+6(24-30)$ & $276^{\circ}$ & $264^{\circ}$ & $12^{\circ}$ \\
\hline $\mathrm{BMJ}+6(24-30)$ & $264^{\circ}$ & $263^{\circ}$ & $1^{\circ}$ \\
\hline $\mathrm{KF}+6(30-36)$ & $288^{\circ}$ & $270^{\circ}$ & $18^{\circ}$ \\
\hline $\mathrm{BMJ}+6(30-36)$ & $276^{\circ}$ & $257^{\circ}$ & $19^{\circ}$ \\
\hline $\mathrm{KF}+6(36-42)$ & $279^{\circ}$ & $270^{\circ}$ & $9^{\circ}$ \\
\hline $\mathrm{BMJ}+6(36-42)$ & $254^{\circ}$ & $246^{\circ}$ & $8^{\circ}$ \\
\hline $\mathrm{KF}+6(42-48)$ & $285^{\circ}$ & $284^{\circ}$ & $1^{\circ}$ \\
\hline $\mathrm{BMJ}+6(42-48)$ & $267^{\circ}$ & $250^{\circ}$ & $17^{\circ}$ \\
\hline $\mathrm{G} 3+6(30-36)$ & $297^{\circ}$ & $282^{\circ}$ & $15^{\circ}$ \\
\hline G3+6_NS $(30-36)$ & $280^{\circ}$ & $266^{\circ}$ & $14^{\circ}$ \\
\hline $\mathrm{G} 3+6(42-48)$ & $287^{\circ}$ & $297^{\circ}$ & $10^{\circ}$ \\
\hline G3+6_NS $(42-48)$ & $278^{\circ}$ & $269^{\circ}$ & $9^{\circ}$ \\
\hline $\mathrm{G} 3+6(60-66)$ & $301^{\circ}$ & $289^{\circ}$ & $12^{\circ}$ \\
\hline G3+6_NS $(60-66)$ & $277^{\circ}$ & $284^{\circ}$ & $7^{\circ}$ \\
\hline
\end{tabular}

1329

1330

1331

1332

1333

1334

1335

1336

1337

1338

1339

1340

1341

1342

1343

1344

1345

1346

1347

1348 
1349 Table 8 The same as Table 6, for the Megi case.

\begin{tabular}{|c|c|c|c|}
\hline $\begin{array}{c}\text { Scheme and analysis } \\
\text { period }\end{array}$ & $\begin{array}{l}\text { Simulated direction } \\
\text { (TC motion) }\end{array}$ & $\begin{array}{c}\text { Diagnosed direction } \\
(W N-1 P V T)\end{array}$ & Disagreement \\
\hline $\mathrm{KF}(42-48)$ & $302^{\circ}$ & $293^{\circ}$ & $9^{\circ}$ \\
\hline BMJ $(42-48)$ & $327^{\circ}$ & $285^{\circ}$ & $42^{\circ}$ \\
\hline $\mathrm{KF}(48-54)$ & $299^{\circ}$ & $286^{\circ}$ & $13^{\circ}$ \\
\hline BMJ $(48-54)$ & $315^{\circ}$ & $286^{\circ}$ & $29^{\circ}$ \\
\hline $\mathrm{KF}(72-78)$ & $275^{\circ}$ & $278^{\circ}$ & $3^{\circ}$ \\
\hline BMJ $(72-78)$ & $241^{\circ}$ & $226^{\circ}$ & $15^{\circ}$ \\
\hline $\mathrm{KF}(96-102)$ & $253^{\circ}$ & $264^{\circ}$ & $11^{\circ}$ \\
\hline BMJ $(96-102)$ & $330^{\circ}$ & $319^{\circ}$ & $11^{\circ}$ \\
\hline $\mathrm{G} 3(48-54)$ & $270^{\circ}$ & $280^{\circ}$ & $10^{\circ}$ \\
\hline G3_NS $(48-54)$ & $302^{\circ}$ & $290^{\circ}$ & $12^{\circ}$ \\
\hline G3 $(54-60)$ & $304^{\circ}$ & $300^{\circ}$ & $4^{\circ}$ \\
\hline G3_NS $(54-60)$ & $289^{\circ}$ & $286^{\circ}$ & $3^{\circ}$ \\
\hline $\mathrm{G} 3(84-90)$ & $295^{\circ}$ & $292^{\circ}$ & $3^{\circ}$ \\
\hline G3_NS (84-90) & $268^{\circ}$ & $273^{\circ}$ & $5^{\circ}$ \\
\hline G3 $(90-96)$ & $297^{\circ}$ & $292^{\circ}$ & $5^{\circ}$ \\
\hline G3_NS $(90-96)$ & $276^{\circ}$ & $277^{\circ}$ & $1^{\circ}$ \\
\hline G3 $(96-102)$ & $291^{\circ}$ & $287^{\circ}$ & $4^{\circ}$ \\
\hline G3_NS $(96-102)$ & $259^{\circ}$ & $257^{\circ}$ & $2^{\circ}$ \\
\hline G3 $(102-108)$ & $311^{\circ}$ & $292^{\circ}$ & $19^{\circ}$ \\
\hline G3_NS $(102-108)$ & $252^{\circ}$ & $257^{\circ}$ & $5^{\circ}$ \\
\hline G3 $(120-126)$ & $28^{\circ}$ & $43^{\circ}$ & $14^{\circ}$ \\
\hline G3 $(138-144)$ & $31^{\circ}$ & $24^{\circ}$ & $7^{\circ}$ \\
\hline
\end{tabular}




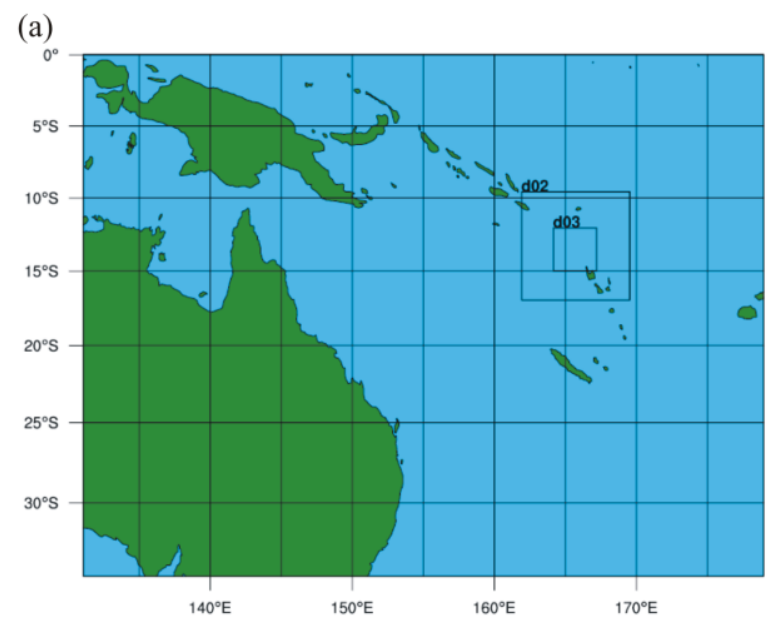

(b)
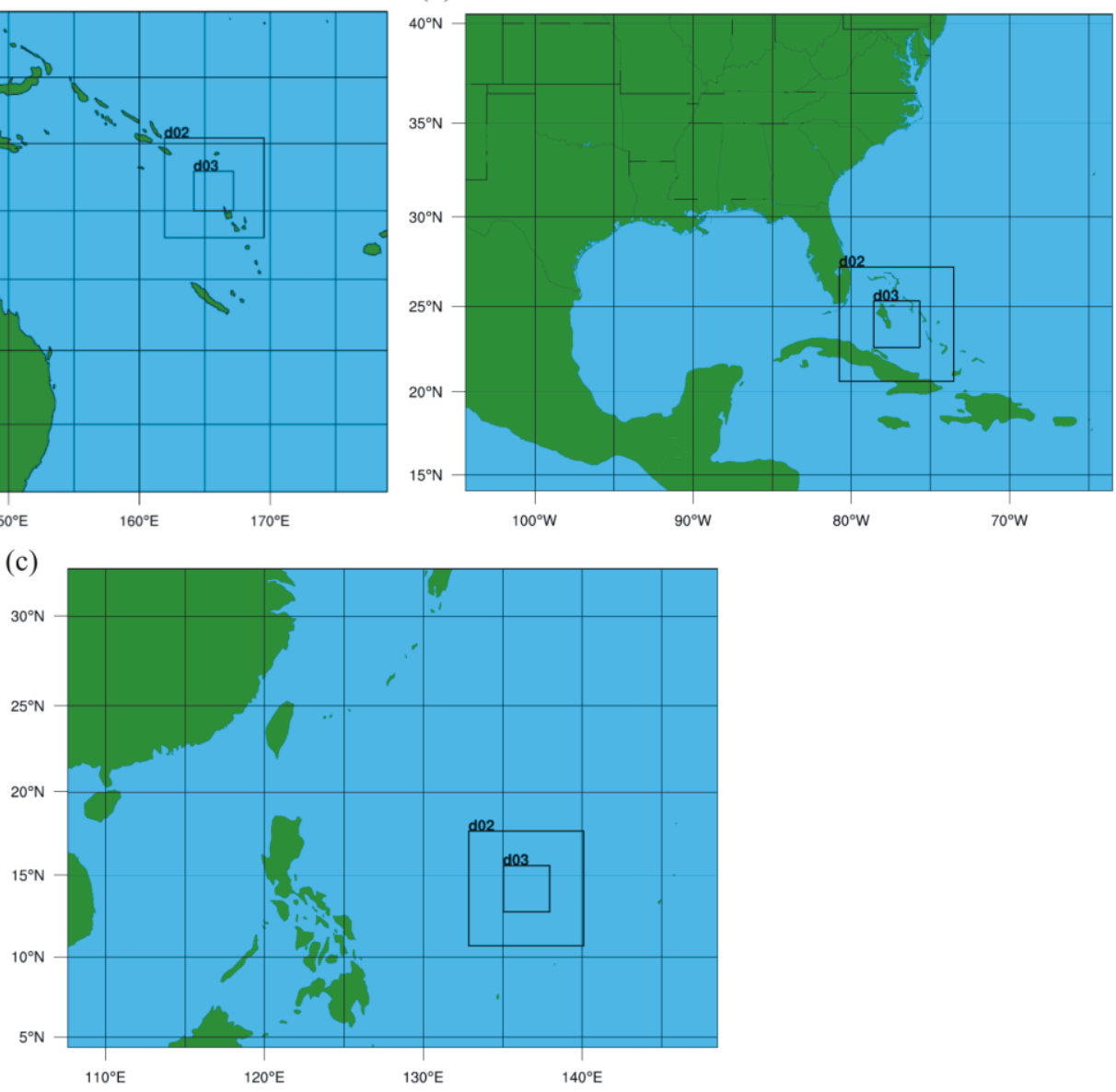

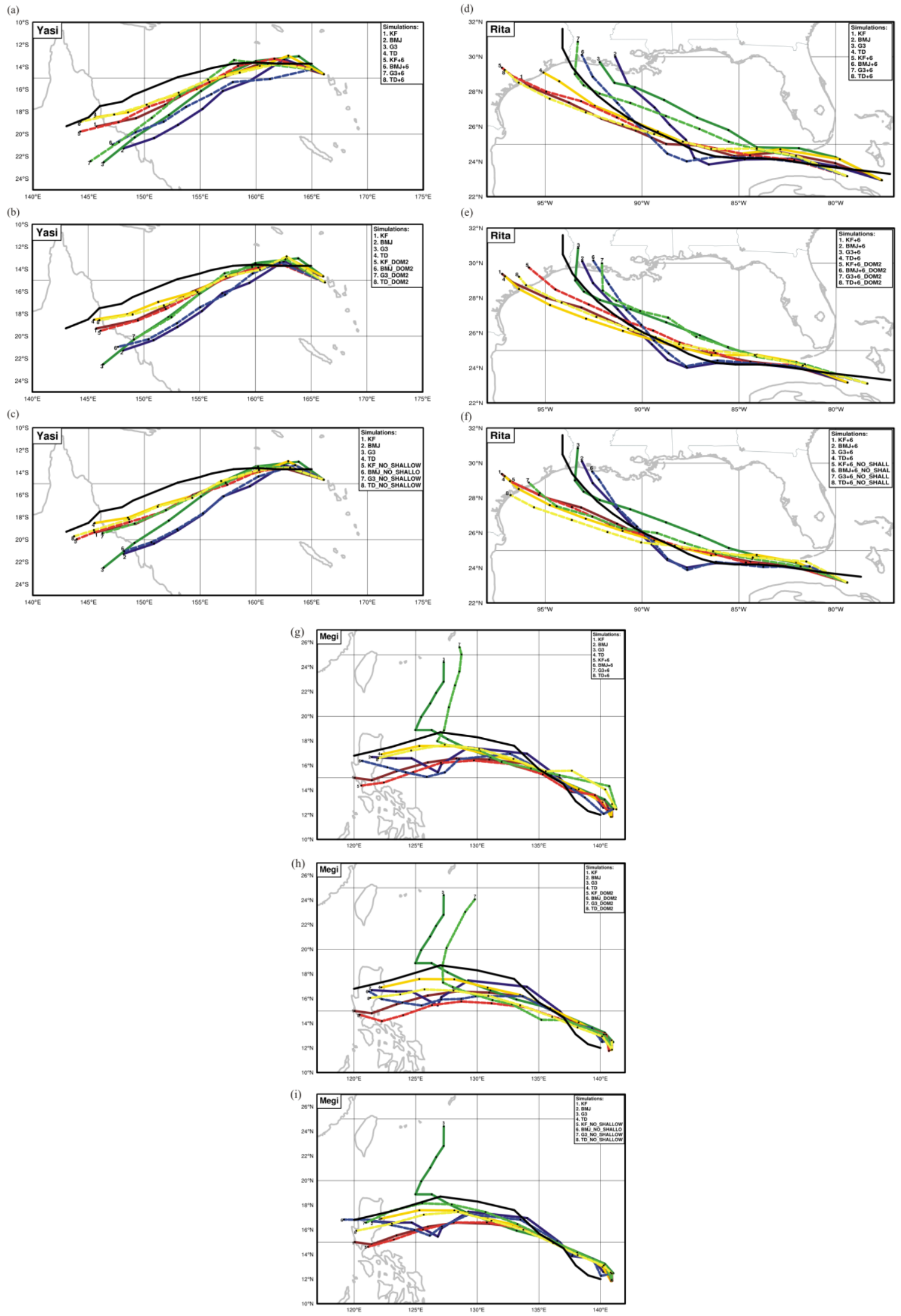

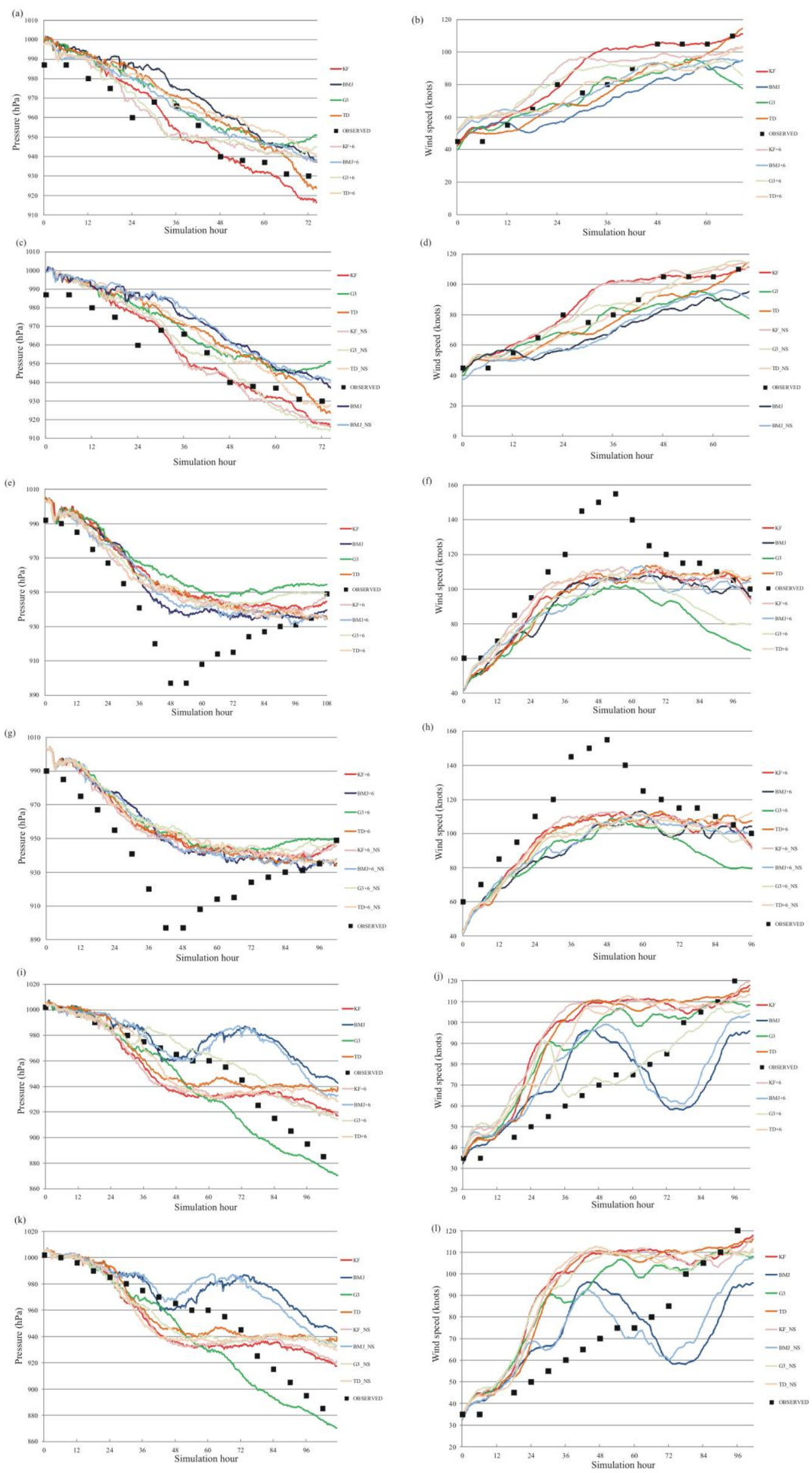
(a)
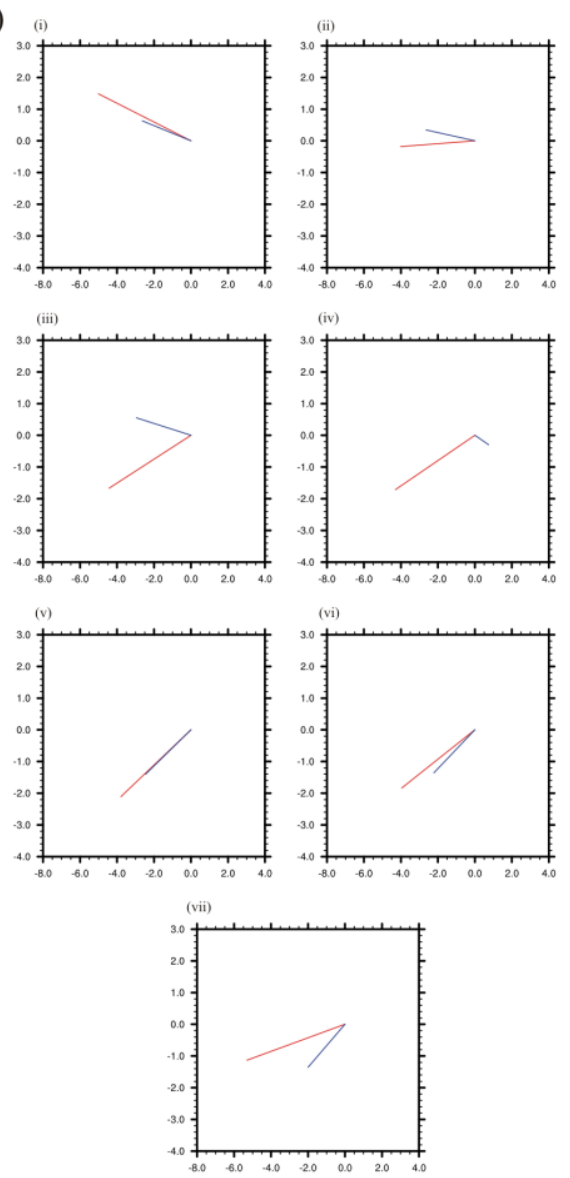

(c)
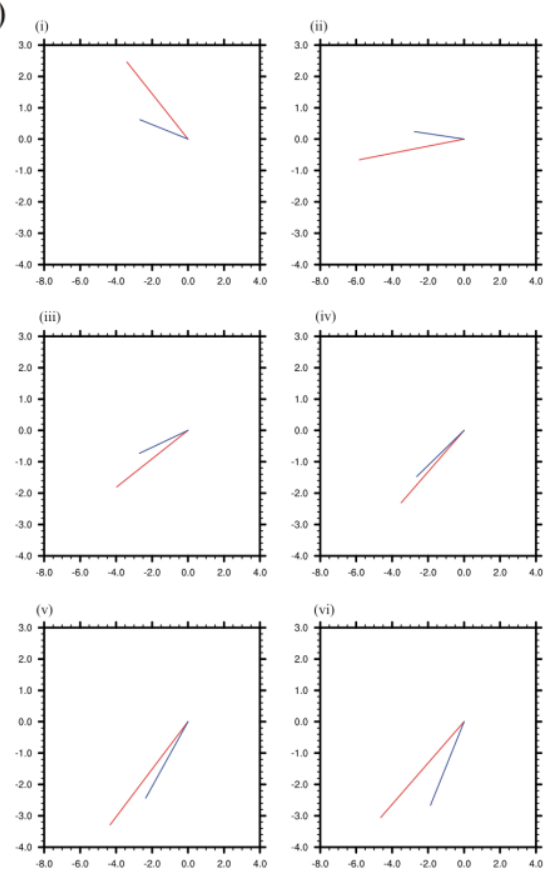

(b)
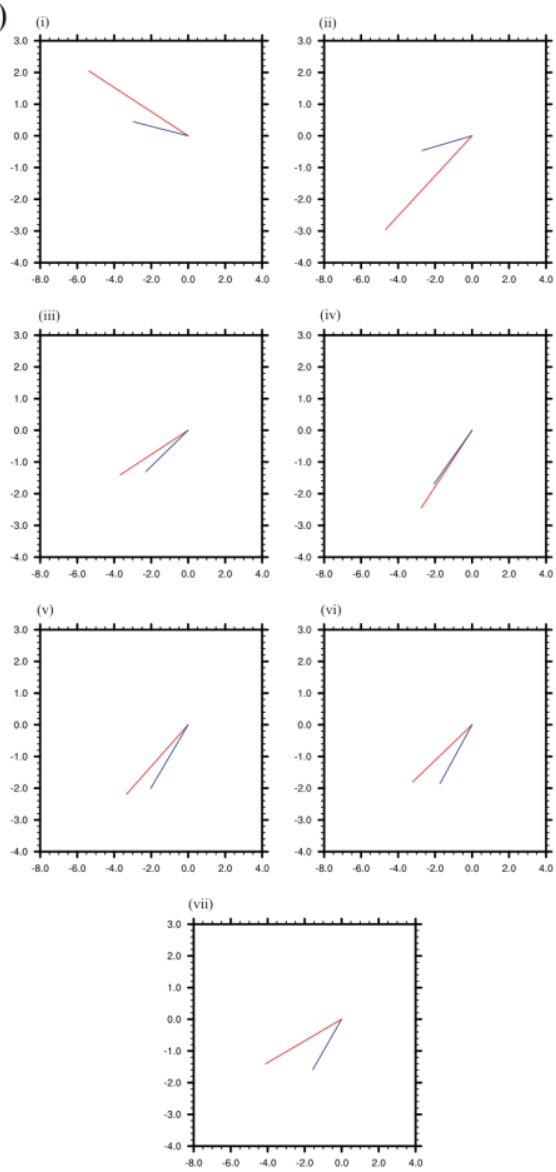

(d)
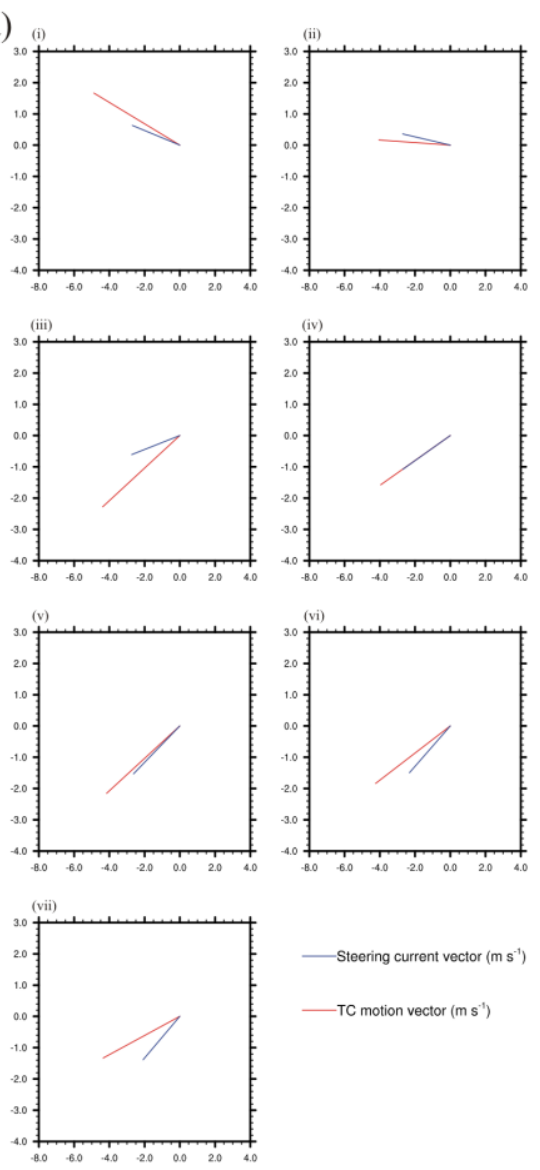


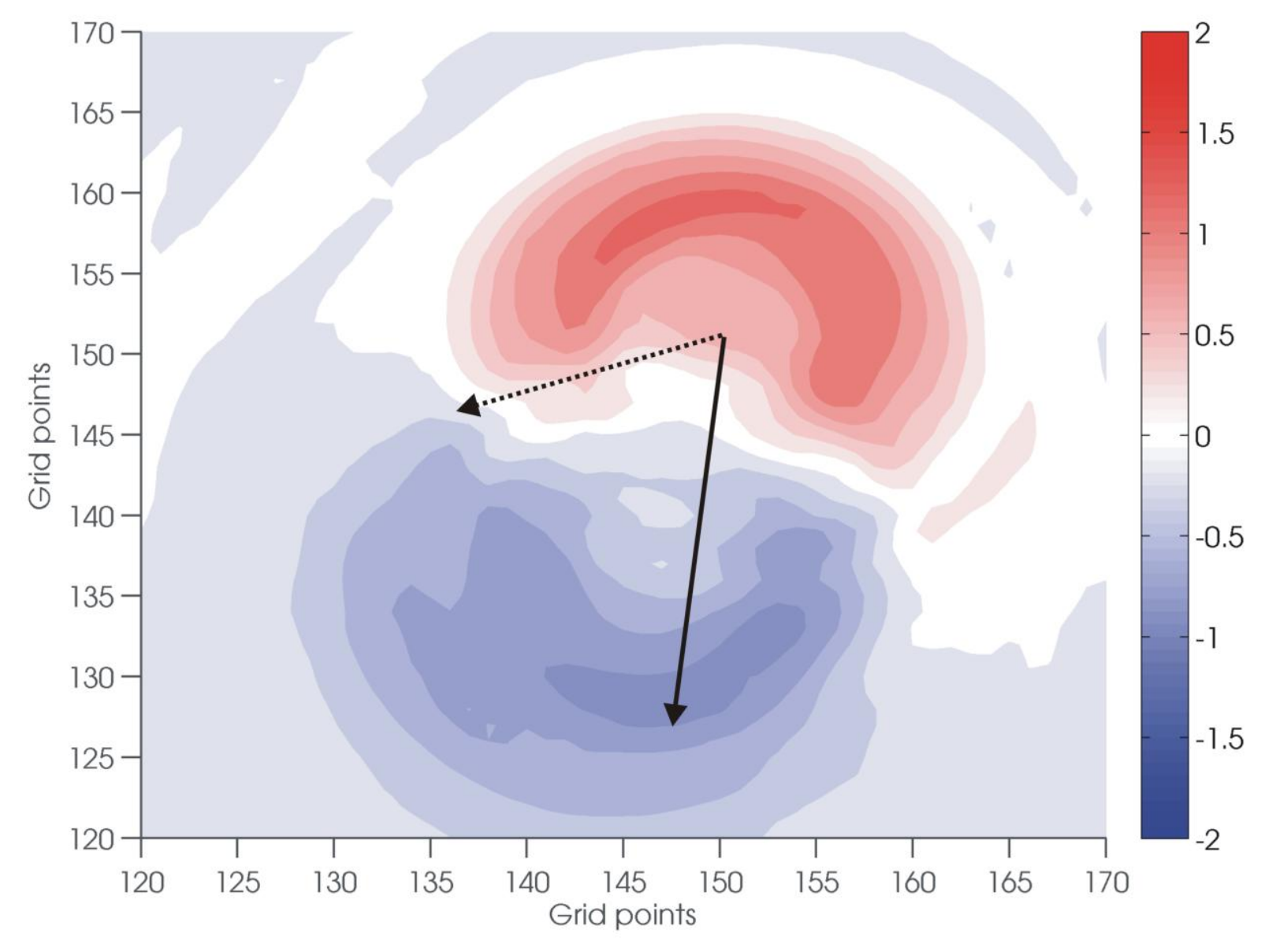

1355 
(a)

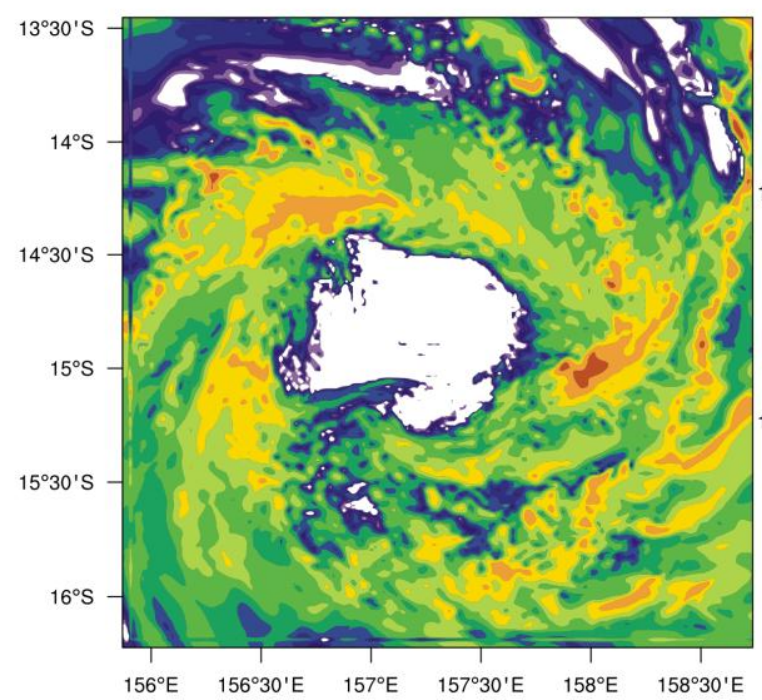

(c)

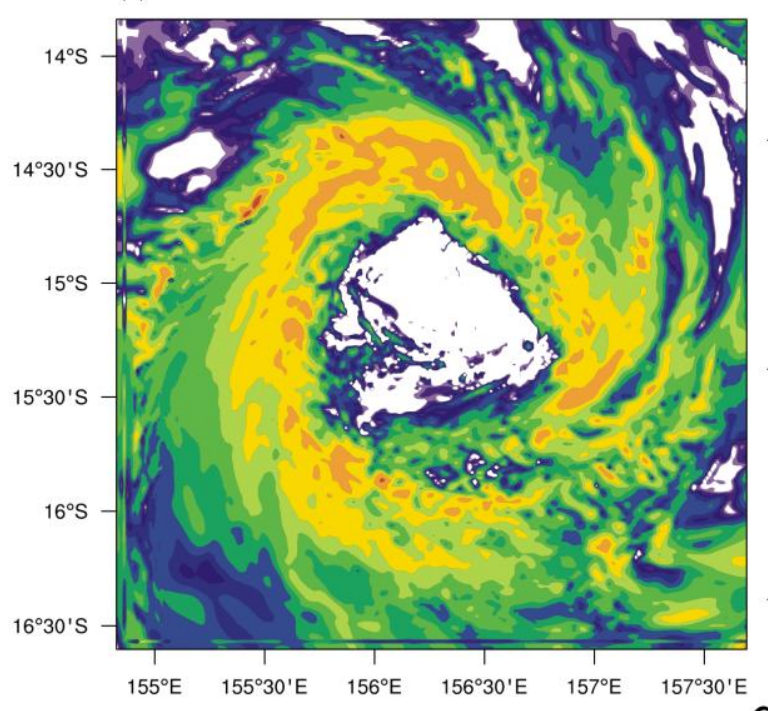

(b)

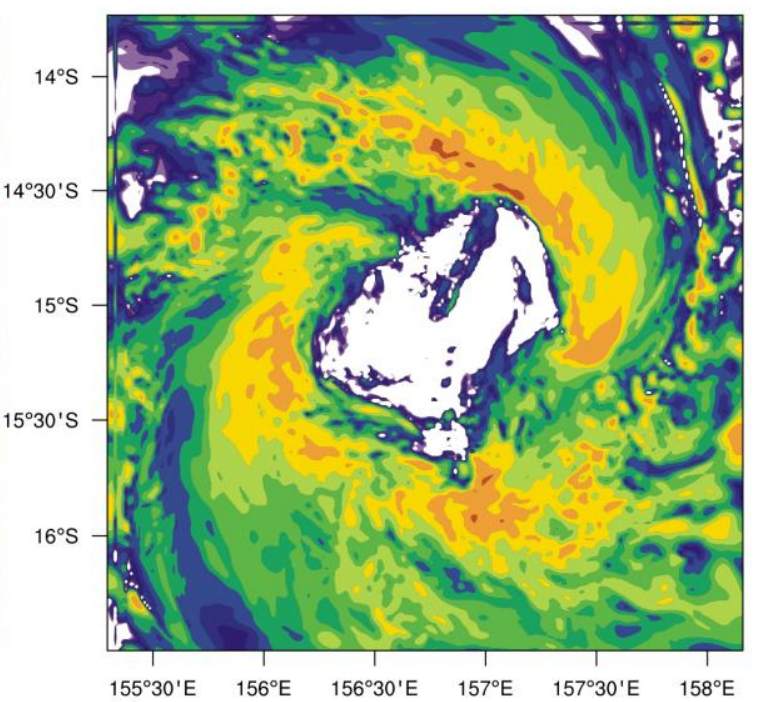

(d)

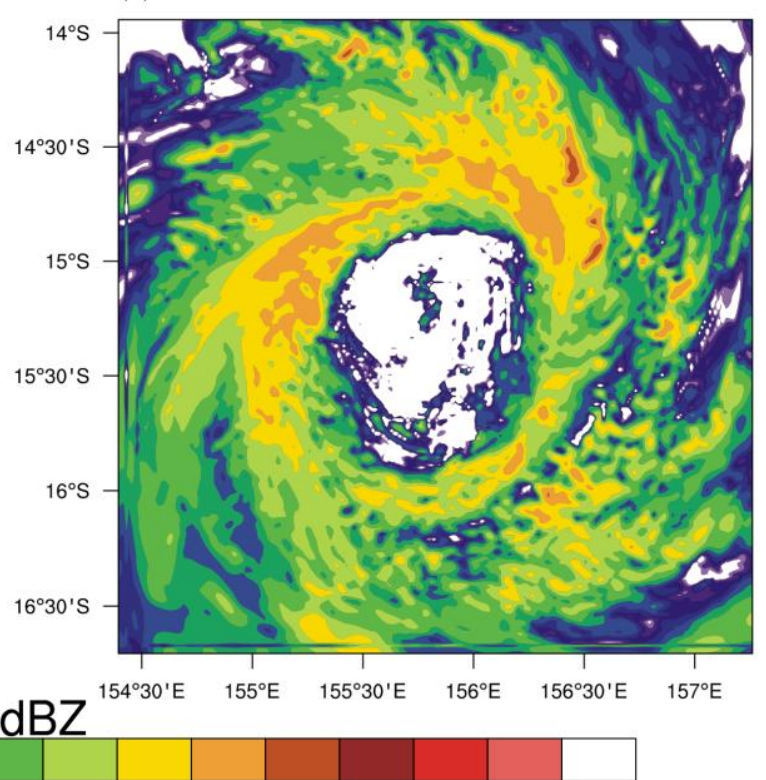

$5 \quad 1015202530354045505560657075$ 
(a)

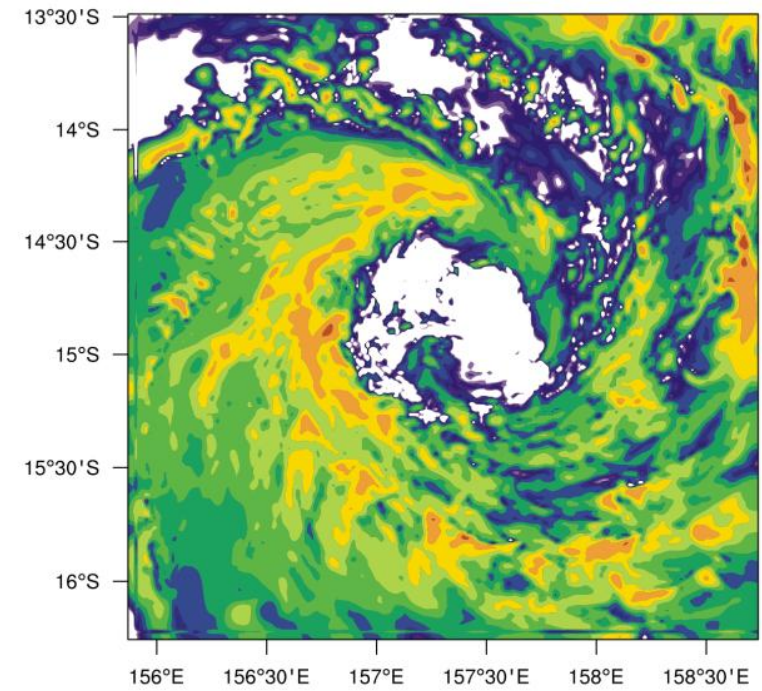

(c)

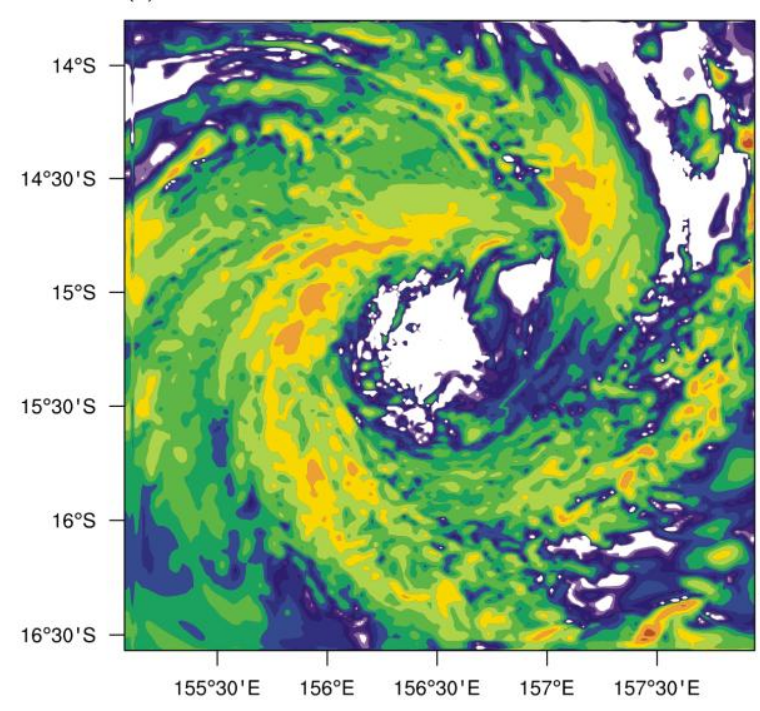

(b)

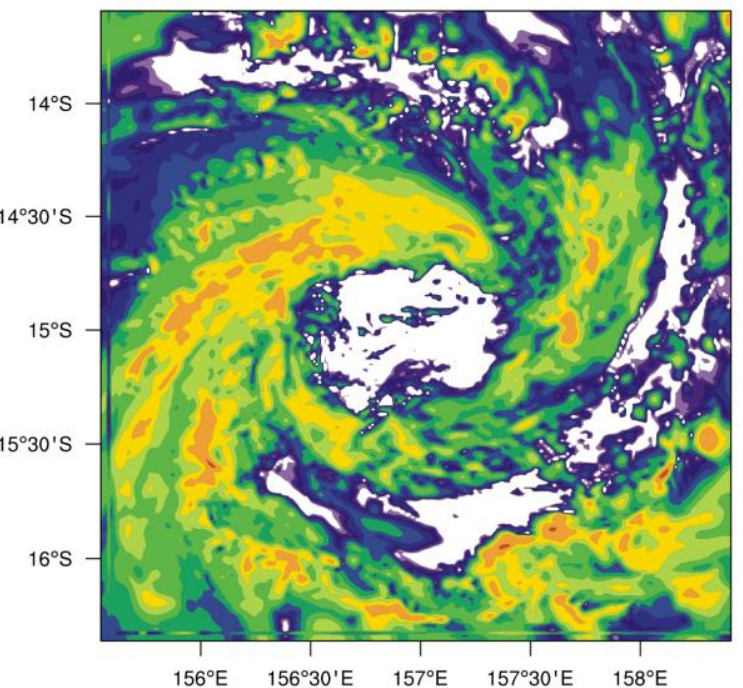

(d)

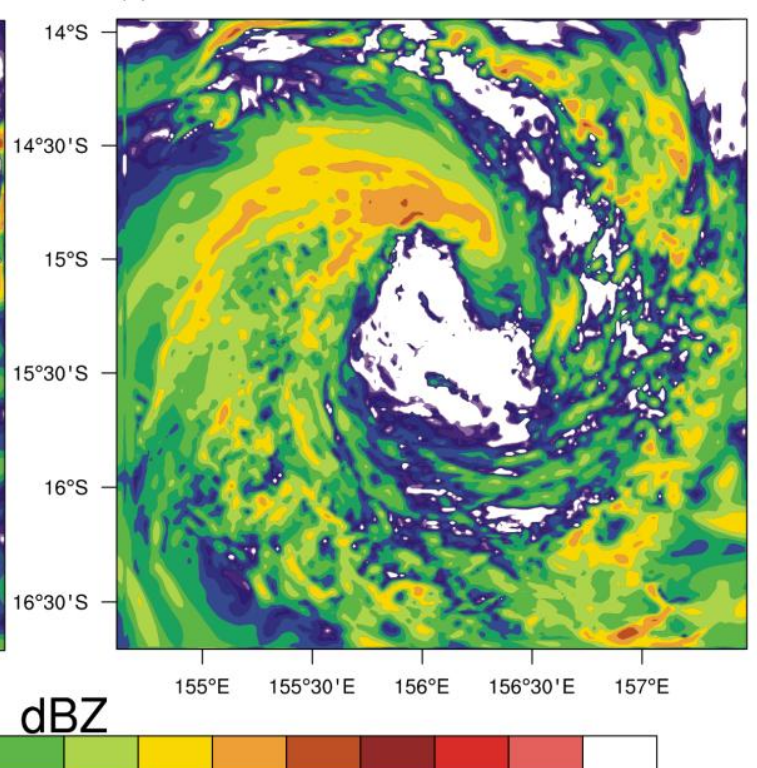

$5 \quad 1015202530354045505560657075$ 
(a)
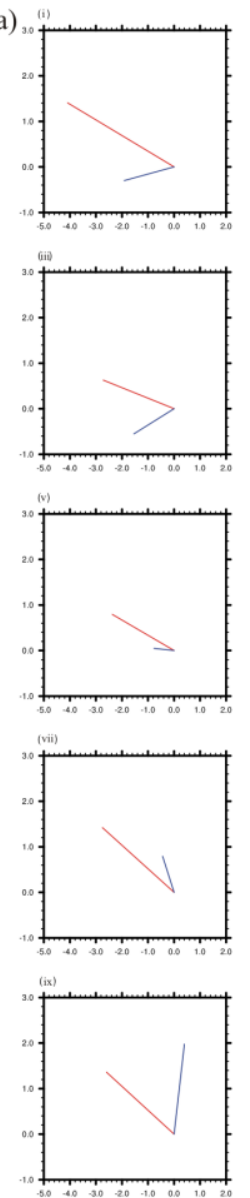

(c)
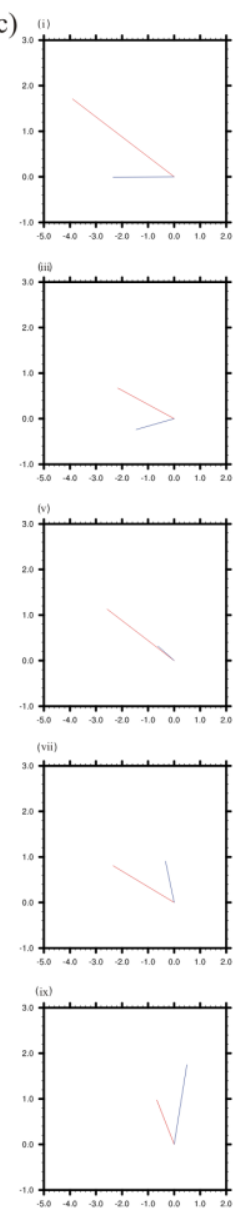

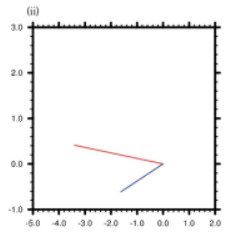

(b)
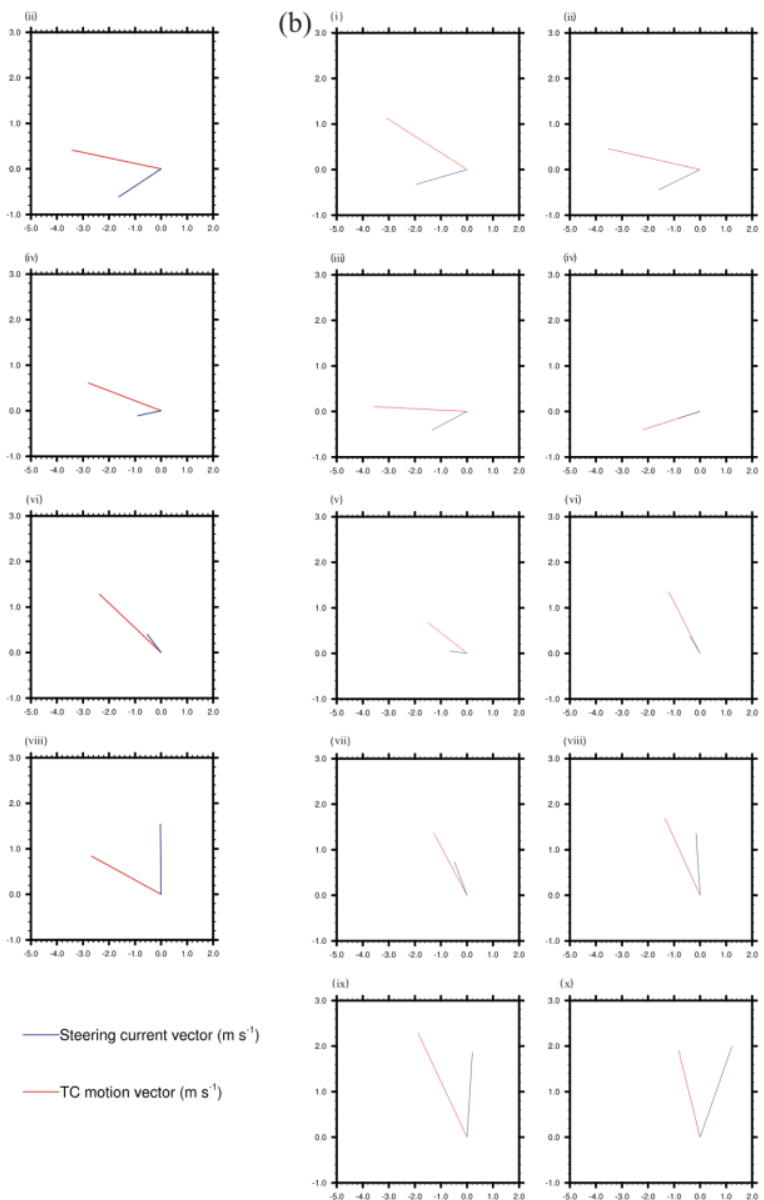

(d) ${ }_{20}$
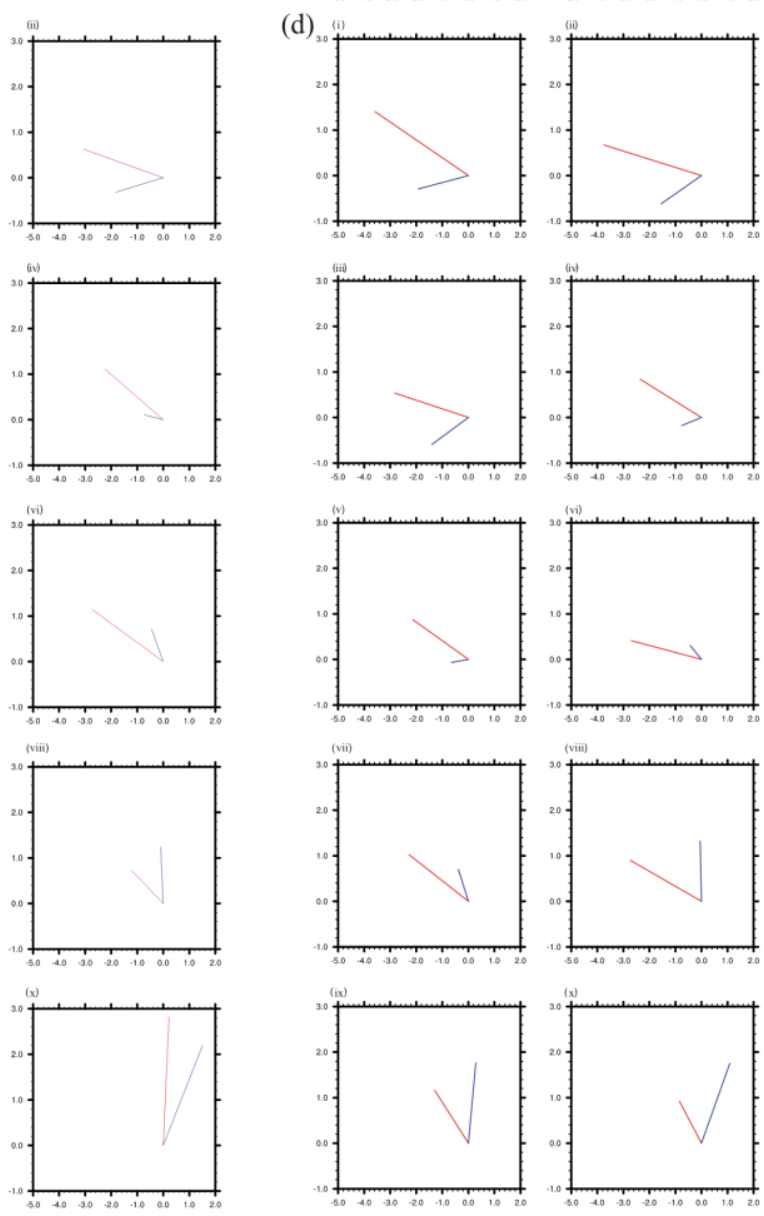
(a)

(b)

1359

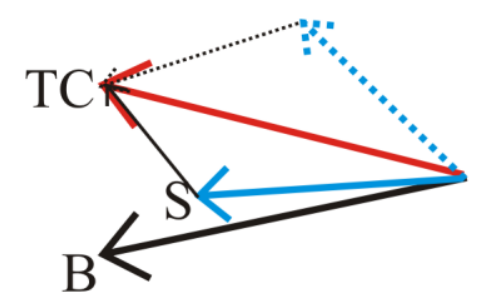

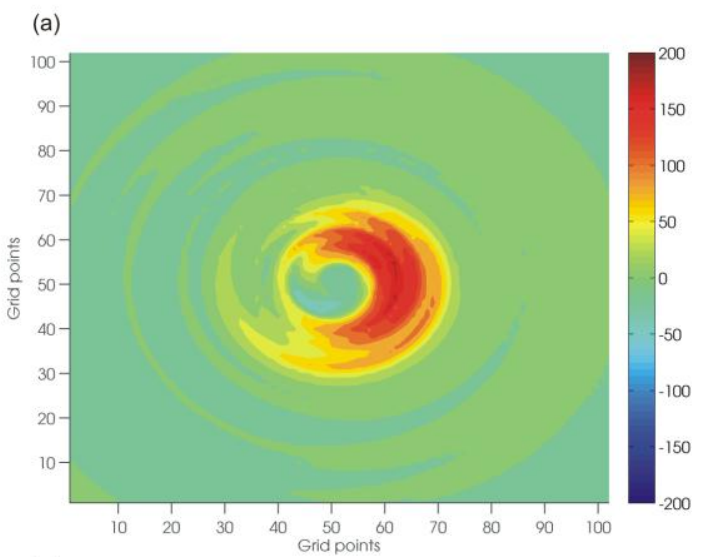

(c)

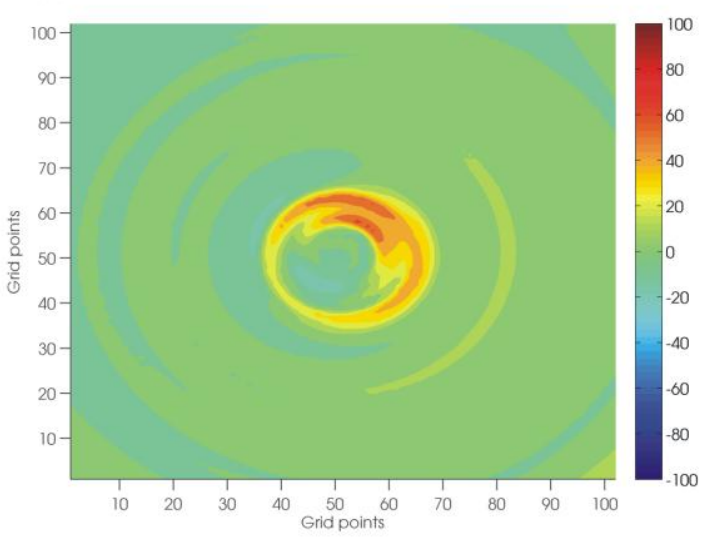

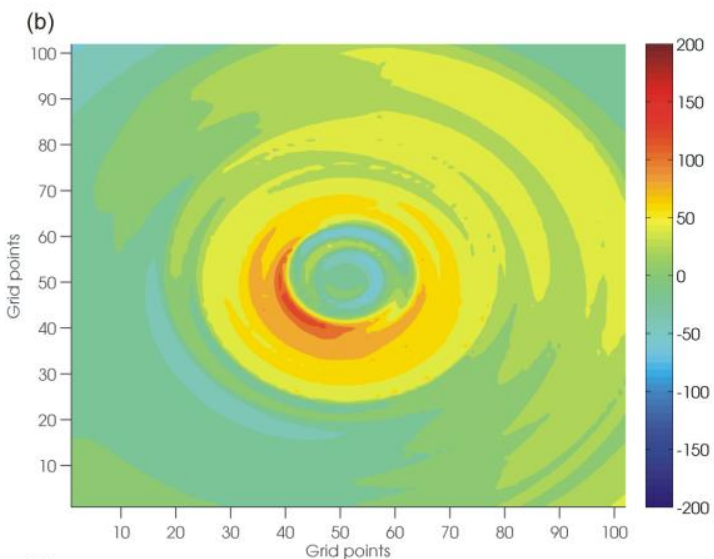

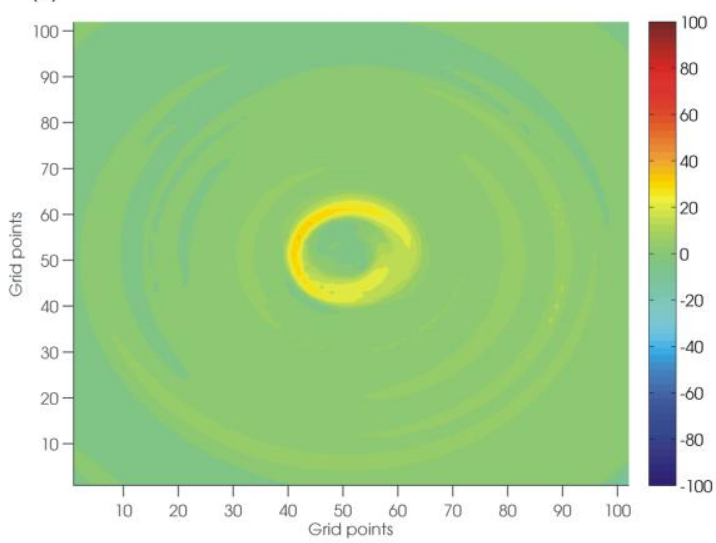


(a)
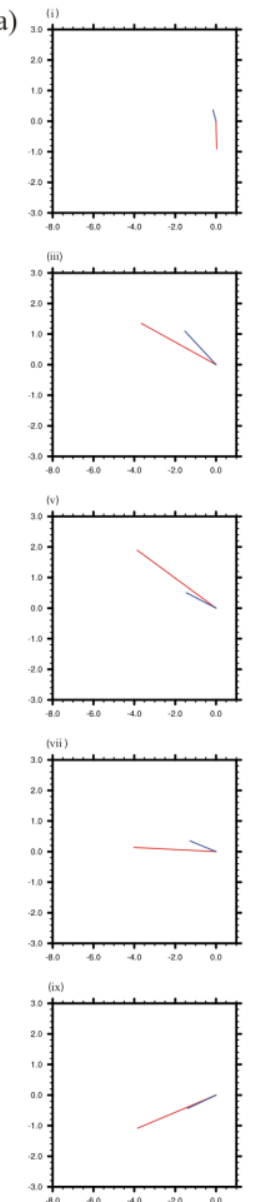

(c)
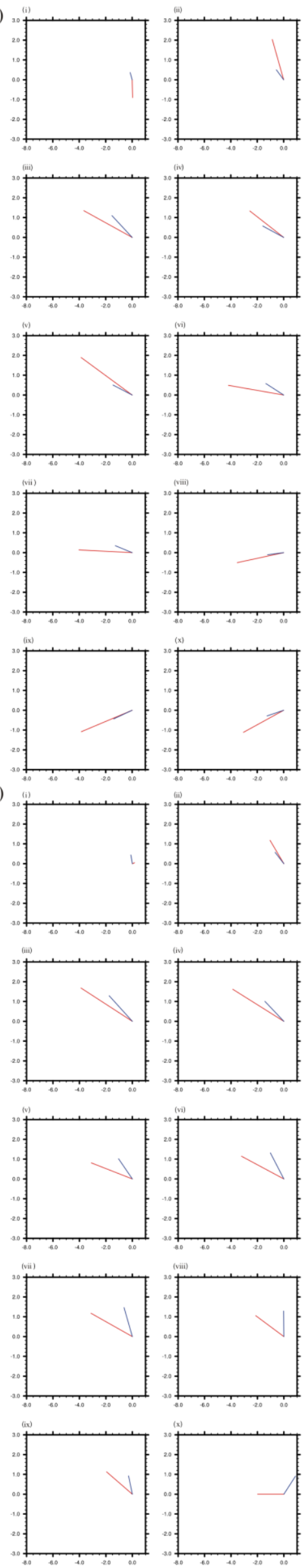

(d)
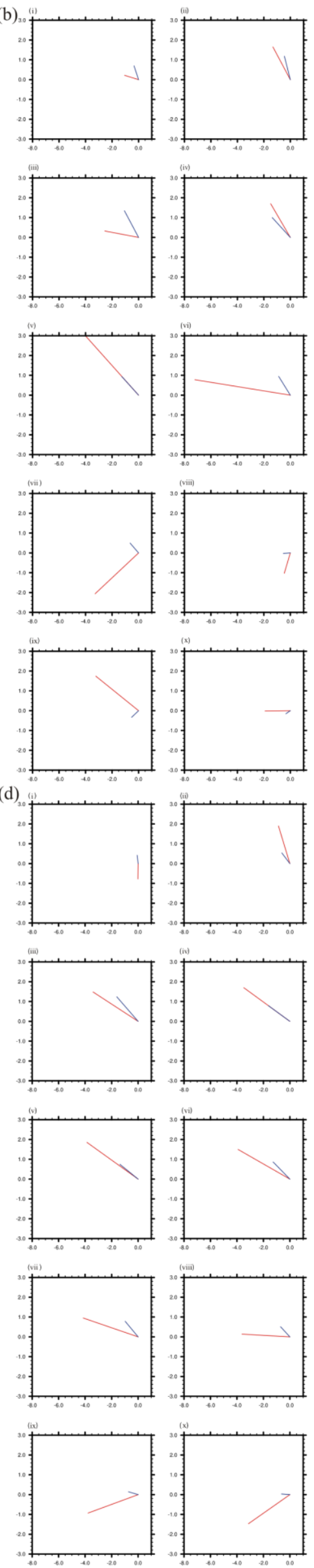
(a)

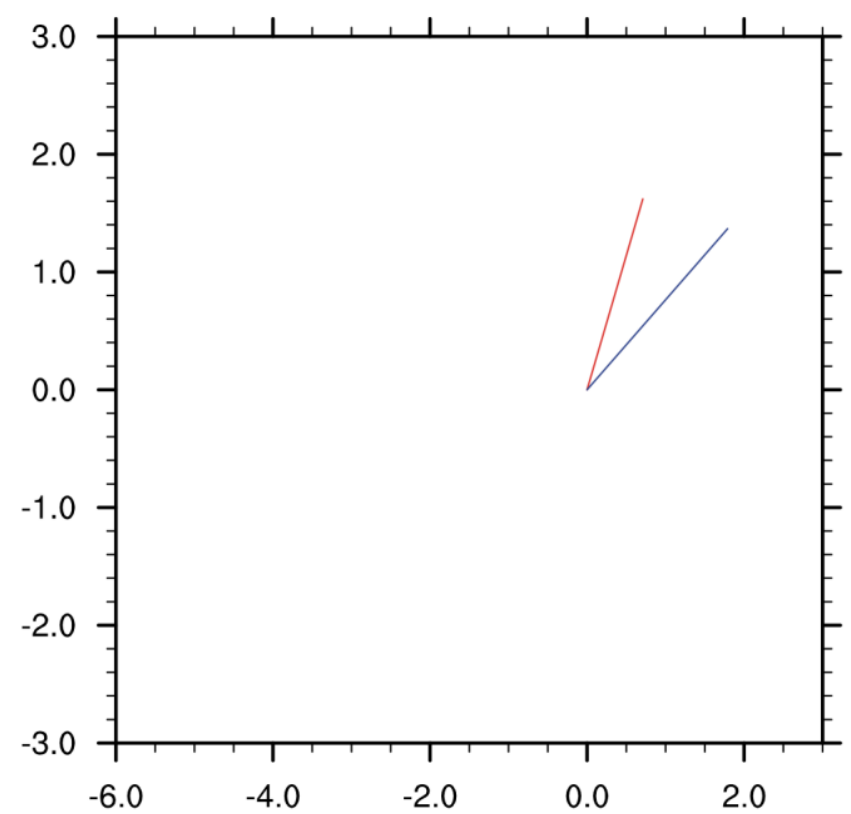

(b)

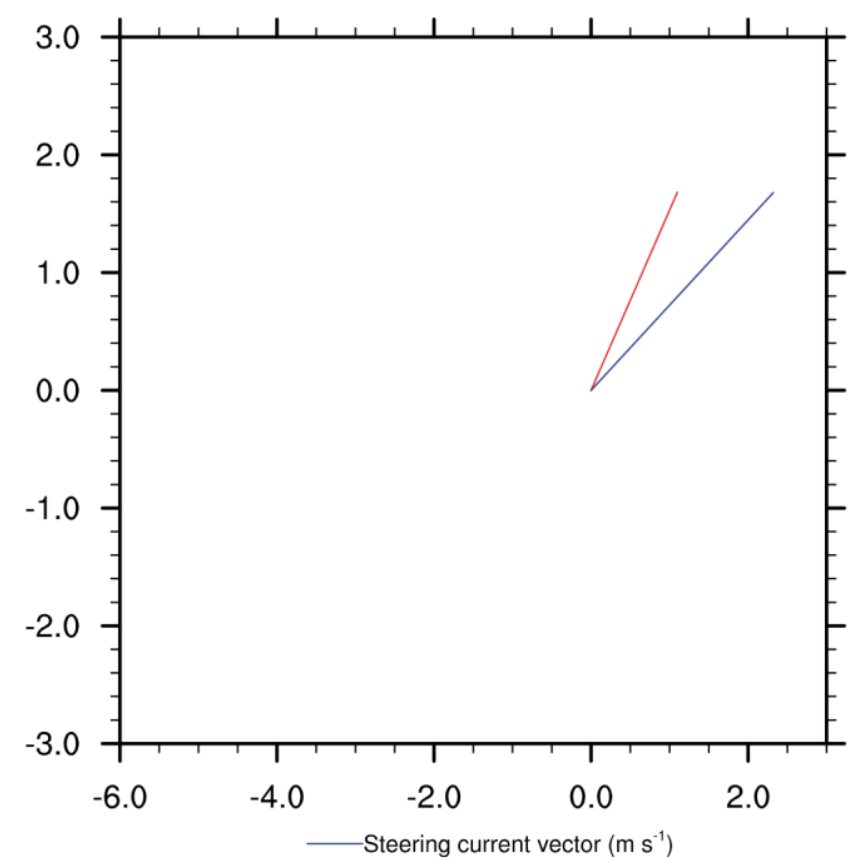


(a)

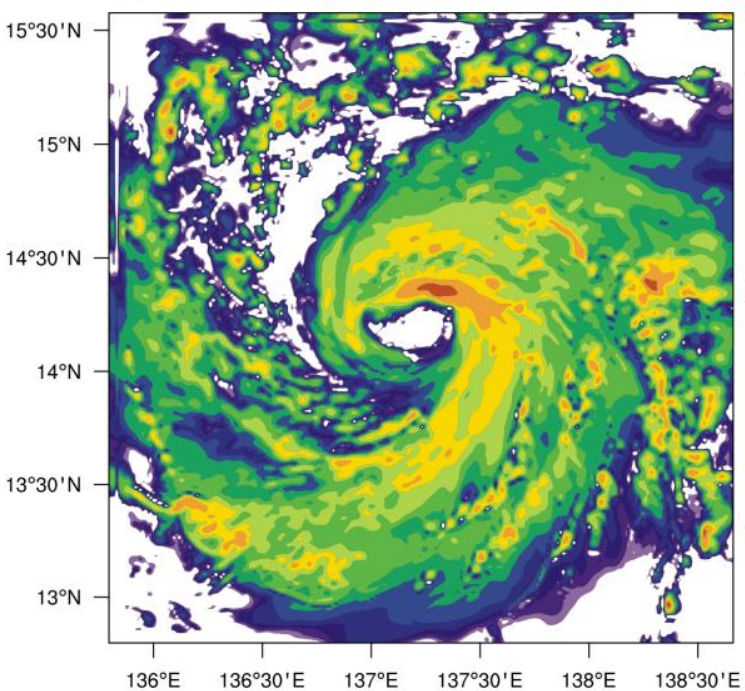

(c)

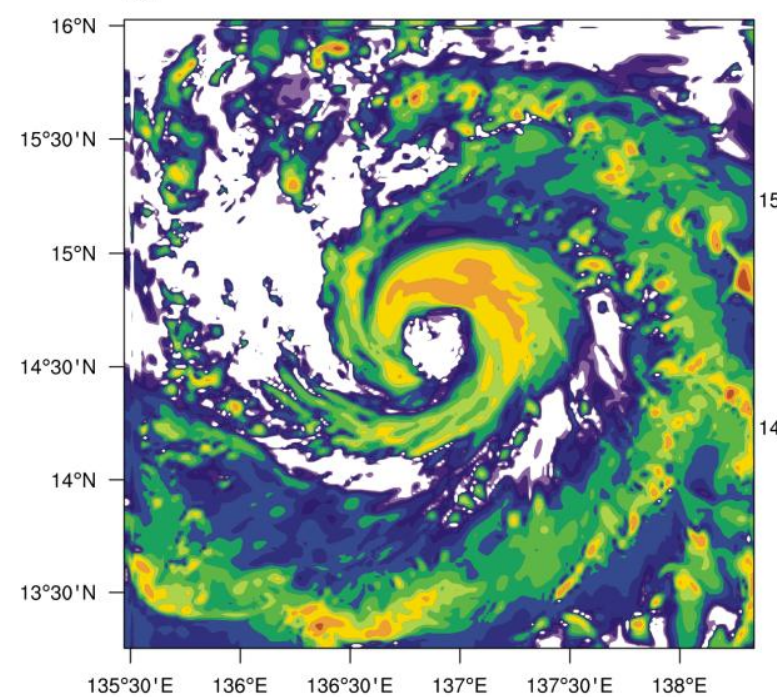

$135^{\circ} 30^{\prime} \mathrm{E} \quad 136^{\circ} \mathrm{E} \quad 136^{\circ} 30^{\prime} \mathrm{E} \quad 137^{\circ} \mathrm{E} \quad 137^{\circ} 30^{\prime} \mathrm{E} \quad 138^{\circ} \mathrm{E}$ (b)

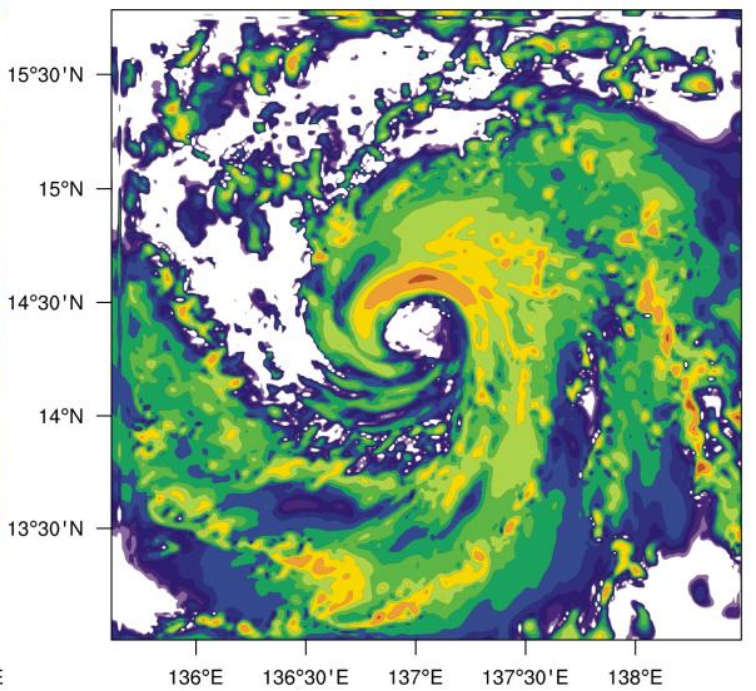

(d)

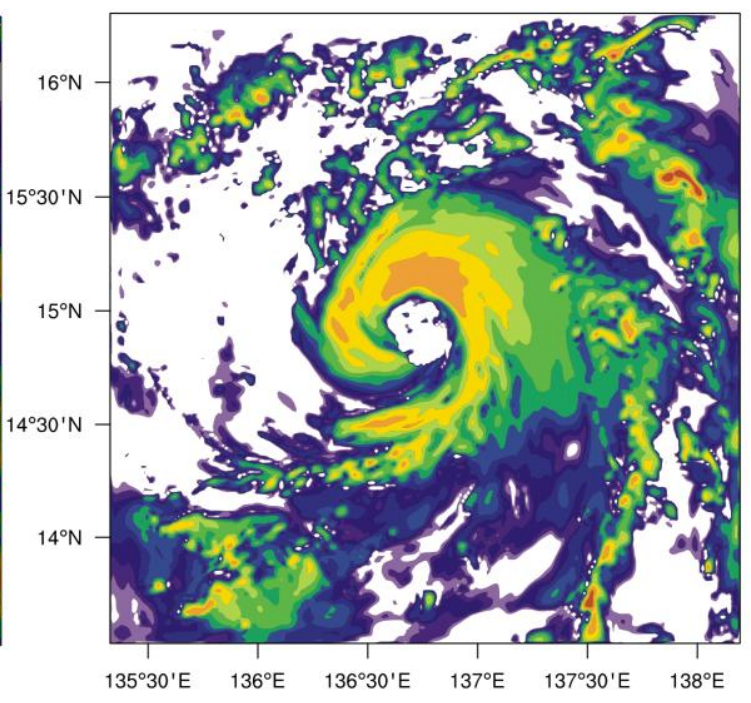

$\mathrm{dBZ}$

$5 \quad 10152025303540 \quad 45505560 \quad 657075$
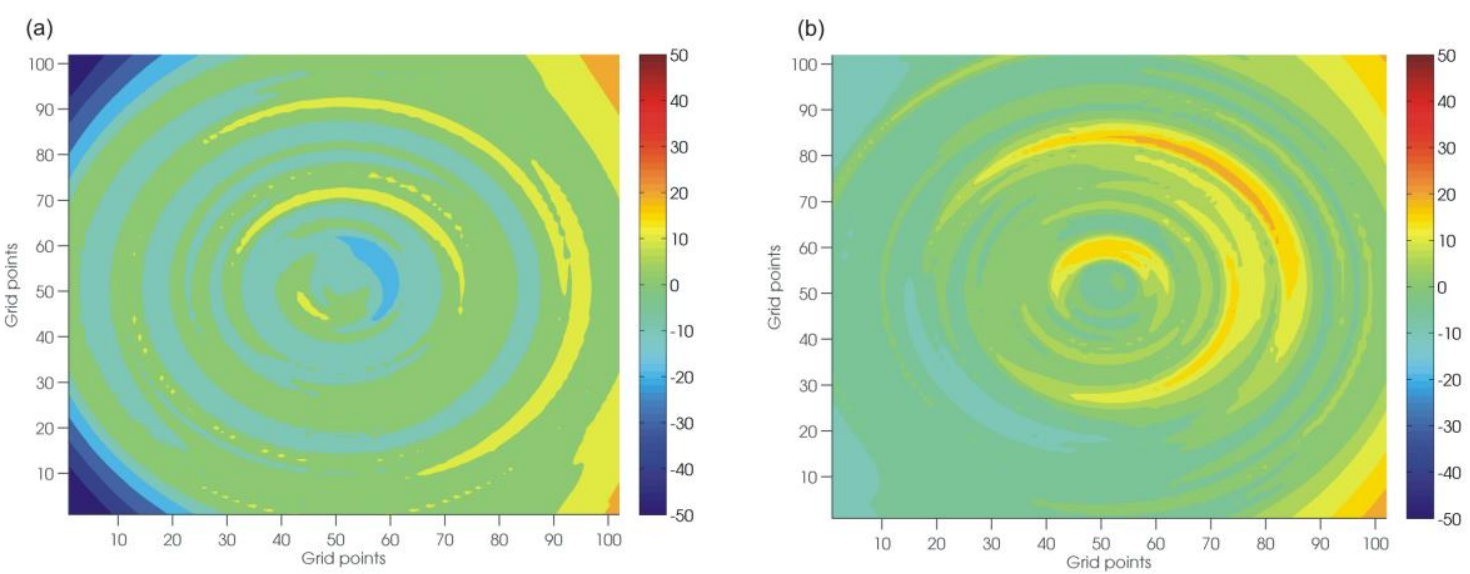


\section{University Library}

\section{- M M I N E R VA \\ A gateway to Melbourne's research publications}

Minerva Access is the Institutional Repository of The University of Melbourne

Author/s:

Shepherd, TJ;Walsh, KJ

Title:

Sensitivity of hurricane track to cumulus parameterization schemes in the WRF model for three intense tropical cyclones: impact of convective asymmetry

Date:

2017-08-01

Citation:

Shepherd, T. J. \& Walsh, K. J. (2017). Sensitivity of hurricane track to cumulus parameterization schemes in the WRF model for three intense tropical cyclones: impact of convective asymmetry. METEOROLOGY AND ATMOSPHERIC PHYSICS, 129 (4), pp.345-374. https://doi.org/10.1007/s00703-016-0472-y.

Persistent Link:

http://hdl.handle.net/11343/282723 